\title{
Trade Policy under Firm-Level Heterogeneity in a Small Economy ${ }^{1}$
}

\author{
Svetlana Demidova ${ }^{2}$ \\ Andrés Rodríguez-Clare ${ }^{3}$ \\ University of Georgia Pennsylvania State University and NBER
}

This version: April 2008

First draft: August 2006

\footnotetext{
${ }^{1}$ We thank Gustavo Ventura, Kala Krishna, Mark Melitz, Jim Tybout, seminar and conference participants at 2007 Midwest International Economics meeting, 2007 North American Summer Meetings of the Econometric Society, 2007 Society for Economic Dynamics Annual Meeting, European Trade Study Group 2007 Athens conference, the University of Iowa and Georgia Institute of Technology for helpful comments and discussions. All remaining errors are our own.

${ }^{2}$ Department of Economics, University of Georgia, Athens, GA 30602. E-mail: demidova@terry.uga.edu. Phone: (706) 542 3689. Fax: (706) 5423376.

${ }^{3}$ Department of Economics, Pennsylvania State University, University Park, PA, 16802. Email: andres@psu.edu. Tel: (814) 861-1295. Fax: (814) 8634775.
} 


\begin{abstract}
We explore the effect of trade policy on productivity and welfare in the now standard model of firm-level heterogeneity and product differentiation with monopolistic competition. To obtain sharp results, we restrict attention to an economy that takes as given the price of imports and the demand schedules for its exports (a "small economy"). We first establish that welfare can be decomposed into four terms: productivity, terms of trade, variety and curvature, where the latter is a term that captures heterogeneity across varieties. We then show how a consumption subsidy, an export tax, or an import tariff allow our small economy to deal with two distortions that we identify and thereby reach its first best allocation. We also show that an export subsidy generates an increase in productivity, but given the negative joint effect on the other three terms (terms of trade, variety, and curvature), welfare falls. In contrast, an import tariff improves welfare in spite of the fact that productivity falls.
\end{abstract}

JEL classification: F12, F13

Key words: trade policies, firm heterogeneity 


\section{Introduction}

Much attention has been devoted recently to the study of international trade when firms differ in terms of productivity. A key contribution is Melitz (2003), which develops a model with firmlevel heterogeneity, product differentiation, increasing returns, monopolistic competition, and fixed as well as variable costs of trade. ${ }^{1}$ Although this has become one of the workhorse models in international trade, little is known about the effects of trade policy in such a setting. In part, this stems from the complexity of the model: trade policy affects welfare through its impact on entry, variety, distortions associated with mark-ups, terms of trade, and home-market effects. In this paper we make headway by restricting attention to a "small economy" that takes as given the unit costs of importers and the demand schedules for its exporters. We find sharp results for the policies that allow the economy to reach its first best allocation and characterize the effects of export subsidies and import tariffs on productivity and welfare.

The economy we consider suffers from two distortions. First, consumers can buy imports at the country's opportunity cost (or international price) but must pay mark-ups on their purchases of domestically produced varieties. This creates a distortion in the allocation of consumers' expenditures between foreign and domestic varieties: there is too little spending on domestic varieties relative to the optimal allocation. We show that a consumption subsidy on domestically produced varieties equal in size to the mark-up neutralizes the first distortion. This can also be accomplished through an import tariff or an export tax of the appropriate magnitude. Second, there is a distortion associated with the fact that an increase in imported varieties increases total consumer surplus, but consumers do not take into account the effect of their spending on imports on the number of imported varieties available for domestic consumption. As a result, the number of foreign varieties available to domestic consumers is below the optimal level. This distortion can be neutralized by a tax on the consumption of domestic varieties, an import subsidy, or an export subsidy.

Optimal values for consumption subsidies, tariffs, or export subsidies can be seen as the combination of the policies that deal with these two distortions. For example, we show that the optimal tariff is the product of the tariff that neutralizes the mark-up distortion and the import subsidy that neutralizes the consumer-surplus distortion. It turns out that the mark-up distortion prevails over the consumption-surplus distortion, so the optimal allocation is attained by the application of either a consumption subsidy, an import tariff, or an export tax.

We also use the model to analyze the effect of export subsidies and import tariffs on productivity and welfare. In models with heterogenous firms, exporters are generally more productive than domestically-oriented firms, so it is conceivable that by reallocating resources from low productivity to high productivity firms, an export subsidy could increase aggregate productivity. This may be the reasoning behind the claim that, by promoting exporting firms rather than those oriented to the domestic market, industrial policy in East-Asian countries was better (or at least less distortionary)

\footnotetext{
${ }^{1}$ See Bernard et. al. (2003) for an alternative formalization of firm-level heterogeneity and international trade.
} 
than in Latin America. Indeed, we show that the positive productivity effect is present: an export subsidy leads to a reallocation of resources from less productive firms oriented to the domestic market to exporters, and this increases overall productivity. Still, since the optimal policy entails an export tax, an export subsidy decreases welfare. To understand why welfare falls in spite of the increase in productivity, we show that welfare can be decomposed into four components: productivity, terms of trade, variety, and curvature, where the latter is a term that captures heterogeneity across varieties. ${ }^{2}$ The negative welfare effect of an export subsidy is associated with its impact on the last three components.

A related paper is Flam and Helpman (1987), which studies the effectiveness of tariffs, export subsidies, and other policies in a model with homogeneous firms. In particular, they develop a model of a small economy with a differentiated good sector (with increasing returns and monopolistic competition) and a sector with constant returns and perfect competition. The existence of this second sector leads to factor price equalization across countries, but it introduces a distortion associated with a difference in the mark-ups across the two sectors. ${ }^{3}$ The authors show that a small tariff is always welfare improving, while the effect of an export subsidy is ambiguous. Our paper follows Flam and Helpman (1987) in focusing on trade policy in a small economy with differentiated goods and monopolistic competition, but adds firm-level heterogeneity and endogenous imported variety as in Melitz (2003). This last element is particularly important in our analysis because it gives rise to the consumer subsidy distortion mentioned above. Additionally, there is an important difference in the way we model a small economy: in Flam and Helpman (1987) firms at Home do not affect the expenditure level in the differentiated good sector abroad, but they can influence the price index there. In our paper, a small economy is "small" in all ways, since Home firms have no effect on both expenditures and the foreign price index. Moreover, we allow the wage to be fully endogenous and affected by trade policy rather than pinning it down through the use of a homogenous good sector.

Another paper related to ours is Baldwin and Forslid (2004), which studies the effects of a decline in the variable and fixed costs of trade on welfare, variety, and productivity. They extend the Melitz (2003) model by looking at two countries that may differ in size and introducing a good produced with constant returns to scale and traded at no cost. This last assumption serves to fix the wage in both countries. In contrast, by focusing on a small country, we can explore how tariffs (and not just trade costs) affect a fully endogenous wage and also characterize optimal policies.

The rest of the paper is organized as follows. The model is laid out and the equilibrium conditions

\footnotetext{
${ }^{2}$ Note that although our economy is "small", its exporters are "big" and set prices. This implies that the terms of trade are not fixed in this economy.

${ }^{3}$ Another paper that also deals with a mark-up distortion in the presence of firm-level heterogeneity, is Bilbiie, Ghironi and Melitz (2006). The authors study the equilibrium of a closed economy, in which there is a distortion caused by the existence of a mark-up for differentiated consumption goods, while there is none for leisure. They show that efficiency can be restored if the government taxes leisure (or subsidizes labor supply) at a rate equal in size to a mark-up charged by producers of differentiated goods. This is analogous to our result that an import tariff or a consumption subsidy neutralizes the mark-up distortion.
} 
are derived in Section 2. Section 3 shows that the first best allocation in the economy can be reached through either a consumption subsidy, an export tax, or an import tariff. Section 4 explores the effects of an export subsidy and an import tariff on the economy. Section 5 concludes. The details of the proofs are given in Appendix.

\section{The Model}

The model incorporates both export and consumption subsidies. Import tariffs can be modeled similarly (see Appendix). Consider a small country with $L$ identical agents. Each agent supplies one unit of labor and spends his income on a continuum of domestic and imported goods indexed by $v$ and $v^{\prime}$, respectively. Domestic and imported goods are consumed in quantities $q(v)$ and $q_{m}\left(v^{\prime}\right)$ by each agent. Preferences are given by

$$
U=\left(\int_{v \in \Omega} q(v)^{\rho} d v+\int_{v^{\prime} \in \Omega_{m}} q_{m}\left(v^{\prime}\right)^{\rho} d v^{\prime}\right)^{1 / \rho}, 0<\rho<1
$$

where $\Omega$ and $\Omega_{m}$ are the sets of available domestic and imported varieties, respectively, and $\sigma=\frac{1}{1-\rho}$ is the elasticity of substitution. We assume that there is a consumption subsidy $1-\eta \geq 0$ for domestic goods, so that consumers pay $\eta p(v)$ given price $p(v)$ charged by producers. Define the price index $P$ by $P^{1-\sigma}=\int_{v^{\prime} \in \Omega_{m}} p_{m}\left(v^{\prime}\right)^{1-\sigma} d v^{\prime}+\int_{v \in \Omega}(\eta p(v))^{1-\sigma} d v$. Then the demand for any variety is:

$$
q(v)=R P^{\sigma-1}(\eta p(v))^{-\sigma} \text { and } q_{m}\left(v^{\prime}\right)=R P^{\sigma-1}\left(p_{m}\left(v^{\prime}\right)\right)^{-\sigma},
$$

where $R$ denotes aggregate expenditure.

Labor is the only factor of production, which is used by a continuum of monopolistically competitive heterogenous firms. Each firm pays a fixed cost $w f_{e}$ to enter the market, where $w$ denotes the wage in the economy. After paying this cost, it derives its productivity draw $\varphi$ according to the cumulative distribution function $G(\varphi)$. To simplify the analysis, we assume that the productivity distribution is Pareto, $G(\varphi)=1-\left(\frac{b}{\varphi}\right)^{\beta}$ for $\varphi \geq b$, with $\beta>\sigma{ }^{4}$

A firm with productivity level $\varphi$ has a labor requirement $f+\frac{q}{\varphi}$ to produce $q$ units of variety $v$ for the domestic market. Thus, it has a marginal cost $\frac{w}{\varphi}$, and given the demand function from (2), it charges a price $\frac{w}{\rho \varphi}$. Then the quantity sold domestically, the revenues, and profits from domestic sales of a firm with productivity $\varphi$ are, respectively,

$$
q_{d}(\varphi)=R P^{\sigma-1}\left(\frac{\eta w}{\rho \varphi}\right)^{-\sigma}, r_{d}(\varphi)=R P^{\sigma-1} \eta^{-\sigma}\left(\frac{w}{\rho \varphi}\right)^{1-\sigma}, \pi_{d}(\varphi)=\frac{r_{d}(\varphi)}{\sigma}-w f
$$

Foreign demand for domestic variety $v$ is given by $A p_{\exp }(v)^{-\sigma}$, where $A$ is exogenously fixed

\footnotetext{
${ }^{4}$ Note that compared to the similar assumption of $\beta>\sigma-1$ in Melitz (2003), we assume $\beta>\sigma$, which allows us to calculate the aggregate quantities produced for the home and foreign markets.
} 
and $p_{\exp }(v)$ is the price charged by an exporter. A firm which decides to export must pay a fixed cost $w f_{\exp }$ to access the foreign market. ${ }^{5}$ Also, we assume that it receives an ad-valorem export subsidy $s>1$, calculated over export revenues, so that an exporter charging price $p_{\exp }$ gets $s p_{\exp }$ for each unit sold abroad. ${ }^{6}$ Thus, exporters maximize

$$
\pi_{\exp }(\varphi)=s A\left(p_{\exp }\right)^{1-\sigma}-(w / \varphi) A\left(p_{\exp }\right)^{-\sigma}-w f_{\exp }
$$

and charge price $p_{\exp }(\varphi)=\frac{w}{s \rho \varphi}$. The quantity exported, the revenues, and profits from exporting are, respectively,

$$
q_{\exp }(\varphi)=A\left(\frac{w}{\rho s \varphi}\right)^{-\sigma}, \quad r_{\exp }(\varphi)=A s^{\sigma}\left(\frac{w}{\rho \varphi}\right)^{1-\sigma}, \quad \pi_{\exp }(\varphi)=\frac{r_{\exp }(\varphi)}{\sigma}-w f_{\exp } .
$$

Since all profits are increasing in $\varphi$, we can define two productivity cutoffs, $x$ and $y$, for domestic producers and exporters, respectively, so that only firms with productivity above $x$ produce for the domestic market, and only firms with productivity above $y$ export. The conditions for these cutoffs are derived from equalizing profits from each option to zero,

$$
\begin{aligned}
R P^{\sigma-1} \eta^{-\sigma}\left(\frac{\rho x}{w}\right)^{\sigma-1} & =\sigma w f \\
(\mathrm{EXP}) \text { condition } A s^{\sigma} w^{1-\sigma}(\rho y)^{\sigma-1} & =\sigma w f_{\mathrm{exp}}
\end{aligned}
$$

We assume parameters are such that $y>x$, i.e., there are some firms that do not export, which is consistent with the empirical evidence. Specifically, firms with $\varphi \in[b, x)$ exit without production, firms with $\varphi \in[x, y)$ produce only for the domestic market, and firms with $\varphi \in[y, \infty)$ produce for both home and foreign markets. Thus, if $M_{e}$ is the mass of entrants and $M$ is the mass of active firms in the economy, then

$$
(1-G(x)) M_{e}=M
$$

i.e., only the share $(1-G(x))$ of entrants survives. ${ }^{7}$ In addition, the mass of exporters is $M_{\exp }=$ $m_{\exp } M$, where $m_{\exp } \equiv \frac{1-G(y)}{1-G(x)}$ is the share of exporters among the whole population of active firms in the economy.

The production structure abroad is similar to that at Home: the productivity distribution of importers is given by $G(\varphi)=1-\left(\frac{b}{\varphi}\right)^{\beta}$ so that the importer with productivity $\varphi$ sets a price $p_{m}=\frac{\gamma}{\rho \varphi}$, where $\gamma$ denotes the marginal costs of production abroad (it includes the foreign wage, marginal costs, possible transport costs, etc. $).{ }^{8}$ Moreover, importers have to pay a fixed cost of

\footnotetext{
${ }^{5}$ Introducing per-unit trade costs would not affect our results, so we chose to leave them out to simplify notation.

${ }^{6}$ Note that to model an export tax $\tau$, it is enough to assume that $s<1$, so that $\tau=1-s$. All derivations are the same for any value of $s$.

${ }^{7}$ Here we deviate from Melitz (2003) by considering a static model, where firms enter, profits are realized, and then all firms exit in a single period.

${ }^{8}$ If the lower limit of the distribution of productivity for the rest of the world differs from that of the home country,
} 
exporting denoted by $F_{\text {exp }}$. Under the assumption of a small economy, both $\gamma$ and $F_{\exp }$ are not affected by any changes in the home country. Moreover, under this assumption, the mass of all available foreign varieties, $M_{\text {Foreign }}$, is also not affected by any changes at Home. However, only some foreign firms become importers. In particular, if we denote by $z$ the productivity level of a marginal firm, which is indifferent between importing and not, then the mass of importers is $M_{m}=(1-G(z)) M_{\text {Foreign }}$. To simplify the analysis, we normalize the mass of all available foreign varieties to one: $M_{\text {Foreign }}=1$. The level of $z$ is determined from the zero profit condition for importers:

$$
r_{m}(z)=R P^{\sigma-1}\left(\frac{\gamma}{\rho z}\right)^{1-\sigma}=\sigma F_{\mathrm{exp}}
$$

Given the structure above, we can rewrite the price index at Home as

$$
P^{1-\sigma}=\theta M_{m}\left(\frac{\rho z}{\gamma}\right)^{\sigma-1}+\theta M\left(\frac{\rho x}{\eta w}\right)^{\sigma-1},
$$

where $\theta \equiv \frac{\beta}{\beta-(\sigma-1)}$. Next, following Melitz (2003), we define $\tilde{\varphi}(x)=\left(\int_{x}^{\infty} \varphi^{\sigma-1} \mu(\varphi) d \varphi\right)^{1 /(\sigma-1)}$, where $\mu(\varphi)=\frac{g(\varphi)}{1-G(x)}=\beta \frac{x^{\beta}}{\varphi^{\beta+1}}$. Then the expected profit from entering is given by

$$
\bar{\pi}=\pi_{d}(\tilde{\varphi}(x))+m_{\exp } \pi_{\exp }(\tilde{\varphi}(y))=w f(\theta-1)+w m_{\exp } f_{\exp }(\theta-1) .
$$

The free entry condition, $\bar{\pi}(1-G(x))=w f_{e}$, can then be written as

$$
\text { (FE) condition } \quad(\theta-1) x^{-\beta}\left[f+m_{\exp } f_{\exp }\right]=\frac{f_{e}}{b^{\beta}} .
$$

Now let us derive the trade balance condition. Total export revenues are $\int_{y}\left(r_{\exp }(\varphi) / s\right) M \mu(\varphi) d \varphi=$ $w f_{\exp } M_{\exp } \sigma \theta / s$, whereas the foreign international value of imports is $\int_{z} r_{m}(\varphi) M_{m} \mu(\varphi) d \varphi=$ $F_{\exp } M_{m} \sigma \theta$. The trade balance condition can then be rewritten as

$$
\text { (TB) condition } M_{m} F_{\exp }=\frac{w}{s} M_{\exp } f_{\exp } \text {. }
$$

We also need to derive the formula for the mass of firms in the economy. Note that the total revenue obtained by domestic producers, $M \sigma\left(\bar{\pi}+w\left(f+m_{\exp } f_{\exp }\right)\right)$, must be equal to $w L .{ }^{9}$ Thus,

$$
\text { (M) condition } \quad M=\frac{L}{\sigma \theta\left(f+m_{\exp } f_{\exp }\right)}=\frac{(\theta-1) b^{\beta} L}{\sigma \theta f_{e}} x^{-\beta}
$$

\footnotetext{
$b$, this will not change our results.

${ }^{9}$ To prove, note that the total expenditure at Home is $R_{m}+R_{d}=V+R_{d}=w L+T=w L-(s-1) V-\frac{1-\eta}{\eta} R_{d}$, where $R_{d}$ and $R_{m}$ are expenditures on the domestic and foreign goods, respectively, and $V$ is the value of exports. This implies $s V+R_{d} / \eta=w L$. Note that while consumers pay $\eta p(\varphi)$, a domestic producer with productivity $\varphi$ receives only $p(\varphi)$. However, while foreign consumers pay $p_{\exp }(\varphi)$, exporters receive $s p_{\exp }(\varphi)$. Thus, the total revenues of domestic firms are $s V+\frac{R_{d}}{\eta}$, which equals $w L$ from above.
} 
where the last equality follows from the (FE) condition.

Finally, we want to simplify the zero profit cutoff condition for importers. Note that the total expenditures are $R=w L+T$, where $T$ is the value of lump sum transfers defined as

$$
T=-(s-1) \int_{y} \frac{r_{\exp }(\varphi)}{s} M \mu(\varphi) d \varphi-(1-\eta) \int_{x} r_{d}(\varphi) M \mu(\varphi) d \varphi .
$$

Normalizing $L$ to 1 , using (5) and (6), and some simplification, we obtain

$$
R=w-w \sigma \theta M\left[\frac{s-1}{s} f_{\exp } m_{\exp }+(1-\eta) f\right] .
$$

Using this expression and (8) in (7), we get (see Appendix):

$$
\text { (z) condition } 1=\sigma \theta M\left[f_{\exp } m_{\exp }+(1-\eta) f+\frac{F_{\exp }}{w}\left(\frac{\gamma}{\eta} \frac{x}{w z}\right)^{\sigma-1}\right] \text {. }
$$

Now we have our equilibrium system of equations (6), (9), (10), (11), and (13) with five unknown variables, $x, y, w, M$, and $z$. We are interested in exploring how different policies affect welfare, which is captured by the utility of the representative consumer. To obtain a useful expression for this utility, we first introduce some definitions. Let $Q_{d}$ and $Q_{m}$ be the total quantity consumed of domestic and imported goods, respectively,

$$
\begin{aligned}
Q_{d} & \equiv M \int_{x} q(\varphi) \mu(\varphi) d \varphi=f(\sigma-1) \frac{\beta}{\beta-\sigma} M x \\
Q_{m} & \equiv M_{m} \int_{z} q_{m}(\varphi) \mu(\varphi) d \varphi=\frac{F_{\exp }}{\gamma}(\sigma-1) \frac{\beta}{\beta-\sigma} M_{m} z
\end{aligned}
$$

and let $Q_{\text {consumed }} \equiv Q_{m}+Q_{d}$ be the total quantity consumed of the imported and domestic goods. Similarly, let $Q_{\exp }$ be the total quantity of goods exported,

$$
Q_{\exp }=M \int_{y} q_{\exp }(\varphi) \mu(\varphi) d \varphi=f_{\exp }(\sigma-1) \frac{\beta}{\beta-\sigma} M_{\exp } y
$$

and let $Q_{\text {produced }} \equiv Q_{\exp }+Q_{d}$ be the total quantity produced for both the domestic and foreign markets. Then the utility per capita can be expressed as

$$
\begin{gathered}
\frac{U}{L}=\frac{Q_{\text {produced }}}{L} \frac{Q_{\text {consumed }}}{Q_{\text {produced }}}\left(M_{t}\right)^{\frac{1}{\sigma-1}} * \\
*\left[\left(\frac{M_{m}}{M_{t}}\right)^{1-\rho}\left(\frac{Q_{m}}{Q_{\text {consumed }}} \frac{M_{m}\left(\int_{z} q_{m}^{\rho}(\varphi) \mu(\varphi) d \varphi\right)^{1 / \rho}}{Q_{m}}\right)^{\rho}+\left(\frac{M}{M_{t}}\right)^{1-\rho}\left(\frac{Q_{d}}{Q_{\text {consumed }}} \frac{M\left(\int_{x} q^{\rho}(\varphi) \mu(\varphi) d \varphi\right)^{1 / \rho}}{Q_{d}}\right)^{\rho}\right]^{1 / \rho}
\end{gathered}
$$

where $M_{t}=M+M_{m}$ is the total variety consumed at Home.

The first component in the product above is the productivity index in the economy measured as 
the total output per worker. ${ }^{10}$ Our productivity measure may seem problematic in that it appears to be summing "apples and oranges". But, in fact, we are adding up quantities that enter the utility function in a symmetric way. So, to continue the metaphor, we are really adding apples of different colors. To see this, forget for a moment about foreign varieties and imagine that "raw quantities" of variety $v$, denoted by $\widetilde{q}(v)$, enter utility as

$$
u=\left(\int_{v \in \Omega}[\lambda(v) \widetilde{q}(v)]^{\rho} d v\right)^{1 / \rho}
$$

Here $\lambda(v)$ could be preference parameters, quality measures, or a way to deal with different ways, in which raw quantities $\widetilde{q}(v)$ could be measured. Let $q(v) \equiv \lambda(v) \widetilde{q}(v)$ denote quantities in "efficiency units". Note that $q(v)$ enters preferences symmetrically across all goods $v$, just as in (1). ${ }^{11}$ Since we are adding up varieties that enter utility symmetrically, then in the present context our productivity measure entails adding up efficiency units, rather than adding up raw quantities (as in $\left.\int_{v \in \Omega} \widetilde{q}(v) d v\right)$. The important point is that our productivity measure is immune to unit changes. A change in units would imply a different function $\lambda(v)$, but leaves $q(v)$ unchanged in the equilibirum.

The second component is the ratio of consumption to production, which is just a trade-adjusted terms of trade (TOT) index. To see this, we can rewrite it as

$$
\text { TOT }=\frac{Q_{\text {consumed }}}{Q_{\text {produced }}}=\frac{Q_{d}+Q_{m}}{Q_{d}+Q_{\exp }}=\frac{P_{\exp }}{P_{m}}\left[\left(\frac{Q_{\exp }}{Q_{d}+Q_{\exp }}\right) /\left(\frac{Q_{m}}{Q_{d}+Q_{m}}\right)\right],
$$

where $P_{\exp }=R_{\exp } / Q_{\exp }$ and $P_{m}=R_{m} / Q_{m}$. In other words, our TOT index takes into account "the importance of trade" in the economy: the ratio of the price of exports to the price of imports (the traditional terms of trade ratio) is multiplied by the ratio of the export share in production to the import share in consumption, which can be treated as a measure of the importance (in quantities) of exports relative to imports. Note that if there is no trade, our TOT index equals 1.

The third component in the utility function is the familiar variety index, and the final component is the curvature term, which includes both within and cross-country heterogeneity. To better understand it, first note that since both countries have the same productivity distributions, then

$$
\frac{M\left(\int_{x} q^{\rho}(\varphi) \mu(\varphi) d \varphi\right)^{1 / \rho}}{Q_{d}}=\frac{M_{m}\left(\int_{z} q_{m}^{\rho}(\varphi) \mu(\varphi) d \varphi\right)^{1 / \rho}}{Q_{m}}=\frac{\beta-\sigma}{\beta}\left(\frac{\beta}{\beta-(\sigma-1)}\right)^{\frac{1}{\rho}}<1 .
$$

This term serves as a measure of heterogeneity among firms within each country. As shown in Appendix, it rises if the dispersion of productivity falls, i.e., if $\beta$ rises. And it converges to 1 as $\beta \rightarrow \infty$. In other words, for any value of $\sigma$, this term becomes closer to 1 as firms differ less, and

\footnotetext{
${ }^{10}$ This definition of productivity differs from that in Melitz (2003), who aims to capture "measured" productivity. In particular, he adds value added across firms and divides this sum by the industry level price, whereas we sum up value added across firms dividing by the price, or $(p q) / p=q$.

${ }^{11}$ Let $\widetilde{\varphi}(v)$ denote the productivity of a producer of good $v$ in raw units. Then the labor productivity of good $v$ in efficiency units is $\varphi(v) \equiv \lambda(v) \widetilde{\varphi}(v)$.
} 
it equals 1 if all firms are identical. Moreover, if $\sigma$ rises then this term rises as well and becomes closer to 1 , which reflects the fact that, with higher elasticity of substitution, differences between varieties and their prices matter less. The curvature term can now be rewritten as

$$
\left[\frac{\beta-\sigma}{\beta}\left(\frac{\beta}{\beta-(\sigma-1)}\right)^{\frac{1}{\rho}}\right] *\left[\left(\frac{M_{m}}{M_{t}}\right)^{1-\rho}\left(\frac{Q_{m}}{Q_{\text {consumed }}}\right)^{\rho}+\left(\frac{M}{M_{t}}\right)^{1-\rho}\left(\frac{Q_{d}}{Q_{\text {consumed }}}\right)^{\rho}\right]^{1 / \rho} .
$$

The second component in the expression above reflects cross country heterogeneity. To see this, note that since each (domestic or foreign) variety enters the utility function symmetrically, in the absence of any heterogeneity in prices within and across countries, households would consume the same quantity of each good so that this term would be equal to $1 .^{12}$

To sum up, there are 4 channels, through which any policy affects welfare in the economy:

$$
\frac{U}{L}=(\text { Productivity Index }) *(\text { TOT index }) *(\text { Variety Index }) *(\text { Curvature })
$$

\section{The First Best Allocation}

Now let us look at the social planner's choice of the optimal policy. ${ }^{13}$ The social planner chooses an allocation that maximizes (1) subject to full employment and balanced trade. Note that while the social planner has full control over domestic firms, this is not so with foreign firms; she can only choose the level of expenditures on the imported goods and in that way affect the variety that foreigners will be willing to import. As shown in Appendix, if there exists a solution to this problem, it is unique. Moreover, we prove the following proposition:

Proposition 1 The first best outcome can be achieved through implementing either one of the following policies:

- a consumption subsidy $1-\eta$, where $\eta=\frac{\beta-\rho}{\beta}<1$;

- an export $\operatorname{tax} \tau=1-s$, where $s=\frac{\beta-\rho}{\beta}<1$;

- an import tariff $t=\frac{\beta}{\beta-\rho}>1$.

\footnotetext{
${ }^{12}$ The adjustment for curvature is independent of anything related to gains from variety. To see this clearly, imagine the simplest model of a closed economy, where the mass of varieties is exogenous and equal to one, so that $v \in[0,1]$ and utility is captured by $u=\left(\int_{0}^{1} q(v)^{\rho} d v\right)^{1 / \rho}$. If productivity is heterogenous, then we can write the decomposition$$
u=\int_{0}^{1} q(v) d v \cdot\left(\left(\int_{0}^{1} q(v)^{\rho} d v\right)^{1 / \rho} / \int_{0}^{1} q(v) d v\right) .
$$

The first term is productivity, while the second term is our adjustment for curvature. Even when there are no variety gains, as in this simple example, our curvature adjustment is needed to convert our productivity measure into something that matters for welfare.

${ }^{13}$ Here we look at the social planner, who maximizes welfare in the small economy only, not in the whole world.
} 
Moreover, any deviation from the optimal policy values reduces welfare monotonically.

Proof. The detailed proof can be found in Appendix. Here we provide a sketch of the proof. First, we derive the system of first order conditions (F.O.C.s) for the social planner's problem. We prove the uniqueness of its solution by showing that if there exist two solutions then they coincide. We next prove the sufficiency of the F.O.C.s by looking at the matrix of second derivatives evaluated at the solution point.

Then we look at the market equilibrium outcomes in the presence of the policies mentioned in this proposition and show that, for the appropriate Lagrangian multipliers in the social planner's problem, the system of F.O.C.s coincides with the market equilibrium conditions for each of the three policies.

Finally, for each policy in the market equilibrium, we show directly that the first derivative of the utility function is negative for any values below the optimal policy levels (for example, for all consumption subsidies with $\eta<(\beta-\rho) / \beta$ ), and it is positive above the optimal values. Thus, the optimal value is the one, for which the first derivative equals zero, and any deviation from it reduces welfare monotonically.

The intuition behind these results is the following. There are two distortions in the economy. First, there is a domestic distortion created by the mark-up: domestic goods are sold at a price above the opportunity cost, whereas imported goods are sold at a price equal to the opportunity cost, so in the equilibrium there is too little consumption of domestic relative to foreign varieties. This distortion is neutralized with a consumption subsidy that allows consumers to pay a price equal to the producer's marginal cost $(\eta=\rho)$. Another way to neutralize this distortion is to set an import tariff, which makes consumers pay the same "mark-up" $\frac{1}{\rho}$ on imported varieties as the one they pay for domestic varieties $\left(t=\frac{1}{\rho}\right)$. Alternatively, by taxing exports $(s=\rho)$, the social planner makes exporting less attractive to producers, so that resources are shifted toward domestic production and the quantity of each consumed variety rises. ${ }^{14}$

The second distortion in the model is related to the fact that foreign producers generate an increase in consumer surplus by their entry into the domestic market. Since consumers do not take into account that their spending on imports increases entry by foreign producers, then the mass of the imported varieties $M_{m}$ is below its optimal value. This distortion can be neutralized by using policies opposite to those in the previous case: now the social planner needs a consumption tax or an export subsidy, $\eta=s=\frac{\beta-\rho}{\beta \rho}$, or an import subsidy, $t=\frac{\beta \rho}{\beta-\rho} \cdot{ }^{15}$

It is interesting to note that as firms become less heterogenous (higher $\beta$ ) then the optimal import subsidy increases (i.e., $t=\frac{\beta \rho}{\beta-\rho}$ falls). Intuitively, the gains from importing additional varieties are larger if the marginal varieties are not too different relative to the ones that are

\footnotetext{
${ }^{14}$ See Helpman (1990) for a discussion of the mark-up distortion in models with monopolistic competiton.

${ }^{15}$ These values for the different policies can be formally derived as the optimal policies in a setting, in which the domestic economy only produces a homogenous good under perfect competition, since in that case the mark-up distortion is not present. Details are available upon request.
} 
already imported (see Arkolakis et. al., 2008). Also, note that with rising elasticity of substitution, both the mark-up and consumer surplus distortions become less severe. The former distortion is smaller since the market power of each firm falls, and the latter distortion decreases because each variety becomes less valuable, so the optimal import subsidy falls (i.e., $t=\frac{\beta \rho}{\beta-\rho}$ increases towards 1).

In the presence of both distortions in the economy, the optimal policy is a product of the two policies needed to neutralize these distortions:

$$
\eta=s=\rho\left(\frac{\beta-\rho}{\beta \rho}\right)=\frac{\beta-\rho}{\beta}<1 \quad \text { and } \quad t=\frac{1}{\rho}\left(\frac{\beta \rho}{\beta-\rho}\right)=\frac{\beta}{\beta-\rho}>1 .
$$

Note that in all cases the mark-up distortion dominates the consumer surplus distortion, so that the resulting policies are a consumption subsidy, an export tax, and an import tariff.

To compare these policies with each other, note that while the "real" values, namely, cutoffs $x$, $y$, and $z$, and masses $M, M_{\exp }$, and $M_{m}$, are the same in each case, the "nominal" values, namely, wage $w$, total revenues $R$, and price index $P$, can differ:

$$
w^{c s}=\frac{\beta}{\beta-\rho} w^{\exp }=w^{m}, \quad R^{c s}=R^{\exp }=\frac{\beta}{\beta-\rho} R^{m}, \quad P^{c s}=P^{\exp }=\frac{\beta}{\beta-\rho} P^{m},
$$

where "cs", "exp", and " $m$ " denote the consumption subsidy, the export tax, and the import tariff cases, respectively.

First, note that the export tax leads to a lower wage compared with the consumption subsidy, but the price index and total revenues are the same. The intuition is that the export tax reduces the demand for labor, since exporting is not such an attractive option anymore, and as a result, the wage is lower in this case. However, price indices are the same, since the prices of the imported varieties are still the same, and the price of any domestic variety is low in both cases either because of the consumption subsidy or the lower wage in the export tax case. The revenues are the same, since in one case the revenues from the export tax compensate for the low labor payments, and in the other case a higher wage compensates for losses due to financing of the consumption subsidy.

Second, wages are the same in the case of a consumption subsidy and an import tariff, however, the price index and revenues are higher in the latter case. The explanation of the higher price index is that consumers have to pay a mark-up on both domestic and imported varieties. However, they have a higher income level thanks to the revenues from the import tariff, and this income allows them to buy the same quantities of every variety as in the case of the consumption subsidy.

In addition, Proposition 1 leads to the following straightforward conclusion:

Corollary 1 In the presence of the optimal consumption subsidy, any trade policy results in welfare losses. 


\section{The Effects of Trade Policies}

In this section, we assume that the government has in place the optimal consumption subsidy (i.e., $\eta=\frac{\beta-\rho}{\beta}$ ) and explore how export subsidies and then import tariffs affect the four major components of the utility function in (18).

\subsection{The Effects of Export Subsidies}

Note from Corollary 1 that an introduction of the export subsidy worsens the equilibrium outcome compared to the case with no subsidy at all. ${ }^{16}$ Moreover, we prove the following result:

Proposition 2 Welfare is monotonically decreasing in the level of the export subsidy.

Proof. In Proposition 1 we already proved this result in the absence of a consumption subsidy. Now the proof is the same except that the optimal value of the subsidy is $s=1$.

To understand better why increasing the export subsidy causes a welfare reduction, we look at the components of the per capita utility function in (18). Before analyzing them, we first look at the effect of the export subsidy on the basic variables in the economy.

Proposition 3 As the export subsidy increases, the productivity cutoff for domestic producers rises, the productivity cutoffs for exporters and importers fall, the wage rises, the mass of entrants remains unchanged, the mass of domestic producers falls, and the masses of exporters and importers increase.

The intuition behind the results is that an increasing export subsidy allows less productive firms to export, so that the cutoff for exporters falls and their mass increases, which leads to similar changes in the characteristics of importers in order to keep trade balance. At the same time, the demand for labor in the economy rises, which leads to a higher wage and makes it harder to produce for the domestic market, so the cutoff for domestic producers rises and their mass falls. These two effects compensate each other so that there is no additional entry as a result.

Now let us look at the productivity index. From (14) and (16), productivity can be written as

$$
\frac{Q_{\text {produced }}}{L}=\frac{Q_{d}+Q_{\exp }}{L}=\frac{\beta(\sigma-1)}{\beta-\sigma} M\left[\frac{f}{L} x+m_{\exp } \frac{f_{\exp }}{L} y\right]
$$

Using the (M) and (FE) conditions, we can rewrite it as

$$
\frac{Q_{\text {produced }}}{L}=\frac{\beta(\sigma-1)}{\sigma \theta(\beta-\sigma)} \frac{f_{e}}{(\theta-1) b^{\beta}}\left[f+f_{\exp }\left(\frac{x}{y}\right)^{\beta-1}\right] x^{\beta+1} .
$$

Since $\beta>\sigma>1$, and $x$ and $\frac{x}{y}$ rise with $s$, the productivity index rises as well:

\footnotetext{
${ }^{16}$ As also follows from Proposition 1 , the export subsidy reduces welfare even in the absence of the consumption subsidy, since the optimal policy is the export tax.
} 
Proposition 4 The productivity index is an increasing function of the export subsidy.

Intuitively, the increasing export subsidy raises the expected profits from exporting, thus, more firms enter the market. Competition becomes more severe and only the most productive firms survive. As a result, labor is reallocated from less to more productive firms, and productivity increases, which is a standard selection effect. ${ }^{17}$ However, from Corollary 1 and Proposition 4 together, it is clear that welfare falls with the export subsidy because the other three components in (18) together fall and more than compensate for the productivity increase. Moreover, it can be shown numerically that depending on the parameters, each of these components can rise or fall with $s .{ }^{18}$

Let us look now at the behavior of the TOT and variety indices closer. It is impossible to make unambiguous predictions about the behavior of these two indices in general. The intuition for this is the following. Consider the TOT index. The export subsidy affects the terms of trade through two channels. The first is the intensive margin, i.e., the export subsidy allows the original exporters to increase the quantity they sell abroad, and this leads to the standard negative effect on the terms of trade. ${ }^{19}$ The second channel is along the extensive margin, as the export subsidy allows more firms to become exporters. As a result, the average productivity of exporters declines and this improves the TOT. The net effect on the TOT is ambiguous.

Now consider the variety index. Since the higher export subsidy results in the exit of the least efficient producers, the mass of domestic varieties falls. However, imported variety rises. Thus, when the costs of exporting are very high and, as a result, the economy imports few foreign varieties, an increase in imported variety can more than compensate for the welfare losses arising from the fall in the domestic variety, as consumers place a relatively high value on imported variety. As a result, the variety index can rise.

\subsection{The Effects of Import Tariffs}

From Proposition 1, we know that the import tariff is an optimal policy in the presence of the two distortions that we have identified in the economy. However, unlike the export subsidy, the import tariff reduces the productivity index. To see this, note that (19) again describes the relationship between the productivity index and the productivity cutoffs for domestic producers and exporters, $x$ and $y$. Since a tariff reduces $x$ and increases $y,{ }^{20}$ the tariff lowers productivity. The intuition for this is that the tariff shifts consumer spending away from imports and towards domestic varieties, and this allows firms with lower productivity levels to break even, also pulling resources away from higher productivity exporters. Thus, as with an export subsidy, a tariff has opposite effects on

\footnotetext{
${ }^{17}$ Note that the productivity index also rises with the export subsidy in the absence of the consumption subsidy.

${ }^{18}$ The details can be found in Appendix.

${ }^{19}$ It can be shown that the price set by the original exporters $p_{\exp }(\varphi)=\frac{w}{s \rho \varphi}$ falls since $w / s$ falls with $s$.

${ }^{20}$ See Appendix for details.
} 
welfare and productivity. ${ }^{21}$

\subsection{Trade Policies and Fixed Costs of Exporting}

Why do the results derived above differ from those in models with homogenous firms and monopolistic competition? There are two key differences: firm heterogeneity and fixed costs of exporting for foreign firms. These two elements imply that imported variety is endogenous, and this is what gives rise to the CS distortion that makes the optimal policy in our setting differ from the one derived in Flam and Helpman (1987), where imported variety is exogenous.

If $F_{\text {exp }}$ converges to zero, then all foreign firms become importers $(z=b)$ and the optimal tariff becomes $1 / \rho{ }^{22}$ This is so even in the presence of firm heterogeneity. This implies that heterogeneity is necessary, but not sufficient for the optimal policy to be different from the one in models with homogenous firms and monopolistic competition.

Similarly, non-zero fixed costs of exporting for foreign firms are necessary, but not sufficient for the CS distortion to arise: as $\beta \rightarrow \infty$ (i.e., as firms become homogenous) all foreign firms become importers $(z=b)$, even in the presence of non-zero fixed costs of exporting, $F_{\exp }>0$. The intuition here is that, for sufficiently high $\beta$, foreign firms become so alike that if one of them can earn non-negative profits from importing to the home country, then all of them can do so.

\section{Conclusion}

Recent research has rightly paid much attention to the role of heterogeneity in productivity across firms. In this paper we have analyzed the impact of this on optimal policy for a small economy in the context of a Melitz-type model. We show that the existence of two distortions, namely, the mark-up distortion and the consumer-surplus distortion, makes it desirable to establish a subsidy on the consumption of domestic varieties, or alternatively an import tariff or an export tax. We also characterize how the size of these interventions depends on the degree of heterogeneity and the elasticity of substitution across varieties.

A particularly interesting result concerns the effect of export subsidies. Trade models with heterogenous firms suggest that export subsidies can indeed increase productivity by inducing a reallocation of labor from less to more productive firms. We have shown in this paper that, with an appropriate measure of productivity, this positive effect is in fact present, but is dominated by the negative effects of the export subsidy on the other determinants of the country's welfare (terms of trade, variety, and curvature). Clearly, an exclusive focus on productivity can be counterproductive: a broader analysis is necessary.

\footnotetext{
${ }^{21} \mathrm{~A}$ tariff also has ambiguous effects on variety and the terms of trade index.

${ }^{22}$ Convergence from the optimal tariff $\frac{\beta}{\beta-\rho}$ derived above (for $z>b$ ) to the optimal tariff $1 / \rho$ (for $z=b$ ) is continuous, since there is a region of "small" values of $F_{\exp }$ for which the optimal tariff takes an intermediate value between $\frac{\beta}{\beta-\rho}$ and $1 / \rho$.
} 
In our model the first best allocation can be achieved by either an import tariff, an export tax or a subsidy to consumption of domestic varieties. The equivalence of these different interventions is due to the simple production structure in our model. For example, if there is also a good produced under constant returns to scale and sold with no mark-up, then clearly the consumption of this good should not be subsidized to achieve optimality. In this case, an import tariff would no longer be a first-best intervention. Instead, a subsidy on both imports and the consumption of domestic varieties (sold with a mark-up) would be called for.

We have focused exclusively on a small economy. How do our results carry over to the case of a large country? Such a country would face the same distortions as a small economy plus two additional ones: the classical terms of trade distortion and the home-market effect. Since both of these elements imply that a tariff would increase the country's welfare, we conjecture that the optimal policy for a large country would also entail a tariff, an export tax, or a subsidy to consumption of domestic varieties. It is important to emphasize, however, that the effect of all these considerations (mark-up, consumer-surplus and terms of trade distortions, and the home-market effect) on an individual country's welfare are different from their effect on worldwide efficiency. For example, although this has not been proven, we conjecture that a model with several identical economies characterized as our small economy above would achieve full efficiency under laissez faire. ${ }^{23}$ Thus, the policies we have identified in this paper are not the ones that should be followed to maximize worldwide efficiency.

\section{References}

[1] Arkolakis, Costas, Svetlana Demidova, Peter J. Klenow, and Andrés Rodríguez-Clare (2008). "The Gains from Trade with Endogenous Variety," forthcoming in American Economic Review Papers and Proceedings.

[2] Baldwin, Richard F., and Rikard Forslid (2004). "Trade Liberalization with Heterogenous Firms," CEPR Discussion Paper 4635.

[3] Bernard, Andrew, Jonathan Eaton, J. Bradford Jensen, and Samuel Kortum (2003). "Plants and Productivity in International Trade," American Economic Review 93(4):1268-1290.

[4] Bilbiie, Florin O., Fabio Ghironi, and Marc J. Melitz (2006). "Monopoly Power and Endogenous Variety in Dynamic Stochastic General Equilibrium: Distortions and Remedies," mimeo.

[5] Flam, Harry, and Elhanan Helpman (1987). "Industrial Policy Under Monopolistic Competition," Journal of International Economics 22:79-102.

\footnotetext{
${ }^{23}$ This is certainly the case when there is no firm-level heterogeneity as in Krugman (1980) and Helpman and Krugman (1989, ch. 7).
} 
[6] Giorgi, Giorgio, Angelo Guerraggio, and J. Thierfelder (2004). "Mathematics of Optimization: Smooth and Nonsmooth Case," Elsevier B.V.

[7] Helpman, Elhanan (1990). "Monopolistic competition in Trade Theory," Special Papers in International Finance 16, International Finance Section, Princeton University Press.

[8] Helpman, Elhanan, and Paul R. Krugman (1989). "Trade Policy and Market Structure," Cambridge MA: The MIT Press.

[9] Krugman, Paul R. (1980). "Scale Economies, Product Differentiation, and the Pattern of Trade," American Economic Review 70:950-959.

[10] Melitz, Mark (2003). "The Impact of Trade on Intra-Industry Reallocations and Aggregate Industry Productivity," Econometrica 71:1695-1725. 


\section{Appendix}

\subsection{Derivation of $(\mathrm{z})$ condition (formula (13) in the paper).}

Using (8) and (TB) condition in (7), we get

$$
\begin{aligned}
R & =\sigma F_{\exp }\left(\frac{\gamma}{\rho z}\right)^{\sigma-1} P^{1-\sigma}=\sigma F_{\exp }\left(\frac{\gamma}{\rho z}\right)^{\sigma-1}\left[\theta M_{m}\left(\frac{\rho z}{\gamma}\right)^{\sigma-1}+\theta M\left(\frac{\rho x}{\eta w}\right)^{\sigma-1}\right] \\
& =\sigma \theta M\left[F_{\exp } \frac{M_{m}}{M}+F_{\exp }\left(\frac{\gamma x}{\eta w z}\right)^{\sigma-1}\right]=\sigma \theta M\left[\frac{w}{s} m_{\exp } f_{\exp }+F_{\exp }\left(\frac{\gamma x}{\eta w z}\right)^{\sigma-1}\right] .
\end{aligned}
$$

Finally, plugging (12) for $R$ and dividing both parts by $w$, we get (z) condition

$$
1=\sigma \theta M\left[\frac{(s-1)}{s} f_{\exp } m_{\exp }+(1-\eta) f+\frac{1}{s} m_{\exp } f_{\exp }+\frac{F_{\exp }}{w}\left(\frac{\gamma x}{\eta w z}\right)^{\sigma-1}\right] .
$$

\subsection{Curvature term}

$$
\frac{M\left(\int_{x} q^{\rho}(\varphi) \mu(\varphi) d \varphi\right)^{1 / \rho}}{Q_{d}}=\frac{M \theta^{1 / \rho} f(\sigma-1) x}{f(\sigma-1) \frac{\beta}{\beta-\sigma} M x}=\frac{\beta-\sigma}{\beta}\left(\frac{\beta}{\beta-(\sigma-1)}\right)^{\frac{\sigma}{\sigma-1}} .
$$

Similarly, $M_{m}\left(\int_{z} q_{m}^{\rho}(\varphi) \mu(\varphi) d \varphi\right)^{1 / \rho} / Q_{m}=\frac{\beta-\sigma}{\beta}\left(\frac{\beta}{\beta-(\sigma-1)}\right)^{\frac{1}{\rho}}$. In the case of the Pareto distribution, $G(\varphi)=1-\left(\frac{b}{\varphi}\right)^{\beta}, \varphi>b, E(\varphi)=\frac{\beta}{\beta-1} b$ and $\operatorname{Var}(\varphi)=b^{2} \frac{\beta}{(\beta-2)(\beta-1)^{2}}$. As a result, if $\beta$ rises, the mean and dispersion fall, and if $\beta \rightarrow \infty$, then $E(\varphi) \rightarrow b$, and $\operatorname{Var}(\varphi) \rightarrow 0$. In other words, an increase in $\beta$ reduces heterogeneity among firms, and if $\beta \rightarrow \infty$, all firms are identical. What happens with (21) in both cases? It rises with $\beta$, since its derivative with respect to $\beta$ is positive:

$$
\frac{\sigma}{\beta^{2}}\left(\frac{\beta}{\beta-(\sigma-1)}\right)^{\frac{\sigma}{\sigma-1}}-\frac{(\beta-\sigma) \sigma}{\beta(\sigma-1)}\left(\frac{\beta}{\beta-(\sigma-1)}\right)^{\frac{1}{\sigma-1}} \frac{\sigma-1}{[\beta-(\sigma-1)]^{2}}=\left(\frac{\beta}{\beta-(\sigma-1)}\right)^{\frac{\sigma}{\sigma-1}+1} \frac{\sigma}{\beta^{3}}>0,
$$

and it converges to 1 as $\beta \rightarrow \infty$. Moreover, if $\sigma$ rises, it falls (given that $\sigma<\beta$ ): $\frac{\beta-\sigma}{\beta}\left(\frac{\beta}{\beta-(\sigma-1)}\right)^{\frac{\sigma}{\sigma-1}}=$ $\left(1-\frac{1}{\beta-(\sigma-1)}\right)\left(\frac{\beta}{\beta-(\sigma-1)}\right)^{\frac{1}{\sigma-1}}$, where the first part falls. The second part falls as well, since $\frac{1}{(\sigma-1)} \ln \left(\frac{\beta}{\beta-(\sigma-1)}\right)$ falls with $\sigma$. What happens if $\sigma \rightarrow \infty$ ? In our model, we have a restriction $\beta>\sigma$. Thus, $\sigma$ is always bounded from above. And if $\sigma \rightarrow \infty$, it means that $\beta \rightarrow \infty$, so that this term $\rightarrow 1$.

\subsection{Proof of Proposition 1}

\subsubsection{Social Planner' Problem and Its Solution.}

Let $q(\varphi)$ be the quantity consumed of a good with productivity index $\varphi$ and let $Q(\varphi)$ be the quantity produced. Then if all varieties $v \in \Omega$ are produced, it must be that $q(v)$ with $v \in \Omega$ maximizes utility 
$\int_{v \in \Omega}^{\infty} q(v)^{\rho} d v$ s.t. $\int_{v \in \Omega}^{\infty}[q(v) / \varphi(v)] d v=K$. This leads to the F.O.C. of $q(v) / q\left(v^{\prime}\right)=\left[\varphi(v) / \varphi\left(v^{\prime}\right)\right]^{\sigma}$. On the other hand, if all varieties $v \in \Omega$ are exported, then it must be that $Q(v)-q(v)$ maximizes export revenue $\int_{v \in \Omega}^{\infty} a(Q(v)-q(v))^{\rho} d v$ s.t. $\int_{v \in \Omega}^{\infty}[(Q(v)-q(v)) / \varphi(v)] d v=J$. This leads to the F.O.C. $\frac{Q(v)-q(v)}{Q\left(v^{\prime}\right)-q\left(v^{\prime}\right)}=\left[\varphi(v) / \varphi\left(v^{\prime}\right)\right]^{\sigma}$. Combining both results, we obtain $Q(v) / Q\left(v^{\prime}\right)=\left[\varphi(v) / \varphi\left(v^{\prime}\right)\right]^{\sigma}$. Thus, an optimal allocation would necessarily have $q(\varphi)=\phi \varphi^{\sigma}$ and $Q(\varphi)-q(\varphi)=\alpha \varphi^{\sigma}$, with $\alpha, \phi>0$ (for the appropriate levels of $\varphi$ ). Moreover, if a variety $v$ with $\varphi(v)$ is consumed (exported), then all varieties with $\varphi>\varphi(v)$ must be consumed (exported).

In addition, if all imported varieties $v \in \Omega_{m}$ are consumed, then it must be that $q_{m}(v)$ with $v \in \Omega_{m}$ maximizes utility $\int_{v \in \Omega_{m}}^{\infty} q_{m}(v)^{\rho} d v$ s.t. $\int_{v \in \Omega_{m}}^{\infty} p_{m}(v) q_{m}(v) d v=K_{m}$, where $K_{m}$ is chosen by social planner. Then $q_{m}(v) / q_{m}\left(v^{\prime}\right)=\left[p_{m}\left(v^{\prime}\right) / p_{m}(v)\right]^{\sigma}$, and an importer with productivity $\varphi$ sets a price $p_{m}(\varphi)=\frac{\gamma}{\rho \varphi}$, where $\gamma$ denotes the marginal costs of production abroad, so that $q_{m}(v) / q_{m}\left(v^{\prime}\right)=$ $\left[\varphi(v) / \varphi\left(v^{\prime}\right)\right]^{\sigma}$. Assume $q_{m}(\varphi)=i \varphi^{\sigma}$. If a variety $v$ with $\varphi(v)$ is imported, then all varieties with $\varphi>\varphi(v)$ must be imported. Let us denote the lowest productivity of the importers by $z$, then it has to satisfy the zero profit condition:

$$
i z^{\sigma} \frac{\gamma}{\rho z}=\sigma F_{\exp }, \quad \text { or } \quad i=\frac{(\sigma-1) F_{\exp }}{\gamma z^{\sigma-1}} .
$$

Thus, we look for an allocation that maximizes welfare, has no goods produced for $\varphi<x$, exports only for goods with $\varphi>y$, subject to full employment and balanced trade:

$$
\begin{gathered}
\max _{x, y, z, M}\left\{\int_{z}^{\infty} q_{m}(\varphi)^{\rho} M_{m} \mu(\varphi) d \varphi+\int_{x}^{\infty} q(\varphi)^{\rho} M \mu(\varphi) d \varphi\right\} \text { s.t. } \\
\int_{x}^{\infty}(f+q(\varphi) / \varphi) M \mu(\varphi) d \varphi+\int_{y}^{\infty}\left(f_{\exp }+\frac{Q(\varphi)-q(\varphi)}{\varphi}\right) M \mu(\varphi) d \varphi+\frac{M f_{e}}{1-G(x)}=1, \\
\int_{y}^{\infty} p_{\exp }(Q(\varphi)-q(\varphi)) M \mu(\varphi) d \varphi=\int_{z}^{\infty} p_{m} q_{m}(\varphi) M_{m} \mu(\varphi) d \varphi
\end{gathered}
$$

where $M_{m}=1-G(z)$. In addition, export revenues are $(Q-q) p_{\exp }$. But $Q-q=A p_{\exp }^{-\sigma}$ implies $a(Q-q)^{-1 / \sigma}=p_{\exp }$, where $a=A^{1 / \sigma}$. Hence, export revenues are $a(Q-q)^{1-1 / \sigma}=a(Q-q)^{\rho}$. Also, recall that we assume the Pareto productivity distribution, $G(\varphi)=1-\left(\frac{b}{\varphi}\right)^{\beta}$. Thus, we have

$$
\begin{gathered}
\max _{x, y, z, M, \alpha, \phi}\left\{M_{m} v^{\rho} \theta z^{\sigma-1}+M \phi^{\rho} \theta x^{\sigma-1}\right\} \\
\text { s.t. } \\
M\left[f+\theta \phi x^{\sigma-1}+m_{\exp } f_{\exp }+m_{\exp } \theta \alpha y^{\sigma-1}+\frac{f_{e}}{b^{\beta}} x^{\beta}\right]=1, \quad \text { and } \quad M_{m} v^{\rho} z^{\sigma-1}=M m_{\exp } a \alpha^{\rho} y^{\sigma-1},
\end{gathered}
$$

where $m_{\exp }=(1-G(y)) /(1-G(x))=(x / y)^{\beta}$. We can rewrite it as

$$
\max _{x, y, z, M, \alpha, \phi}\left\{\left(\frac{(\sigma-1) F_{\exp }}{\gamma}\right)^{\rho} b^{\beta} z^{\rho-\beta}+M \phi^{\rho} x^{\sigma-1}\right\} \quad \text { s.t. }
$$


$M\left[f+\theta \phi x^{\sigma-1}+m_{\exp } f_{\exp }+m_{\exp } \theta \alpha y^{\sigma-1}+\frac{f_{e}}{b^{\beta}} x^{\beta}\right]=1, \quad$ and $\sigma F_{\exp } b^{\beta} z^{-\beta}=M m_{\exp } a \alpha^{\rho} y^{\sigma-1}$,

The Lagrangian is then:

$$
\begin{aligned}
£= & \left(\frac{(\sigma-1) F_{\exp }}{\gamma}\right)^{\rho} b^{\beta} z^{\rho-\beta}+M \phi^{\rho} x^{\sigma-1}+\zeta\left(M m_{\exp } a \alpha^{\rho} y^{\sigma-1}-\sigma F_{\exp } b^{\beta} z^{-\beta}\right) \\
& -\lambda\left(M f+M \theta \phi x^{\sigma-1}+M m_{\exp } f_{\exp }+M m_{\exp } \theta \alpha y^{\sigma-1}+\frac{M f_{e}}{b^{\beta}} x^{\beta}-1\right) .
\end{aligned}
$$

This must be maximized with respect to $z, x, y, \alpha, M$. Letting $h(v)=g(v) /[1-G(v)]$, then:

$(z): \quad \partial £ / \partial z=(\rho-\beta)\left(\frac{(\sigma-1) F_{\exp }}{\gamma}\right)^{\rho} b^{\beta} z^{\rho-\beta-1}+\zeta \beta \sigma F_{\exp } b^{\beta} z^{-\beta-1}=0$,

$(x): \quad \partial £ / \partial x=M \phi^{\rho} \theta(\sigma-1) x^{\sigma-2}-\lambda M(\sigma-1) \theta \phi x^{\sigma-2}-\lambda M m_{\exp } f_{\exp } h(x)-\lambda M m_{\exp } h(x) \theta \alpha y^{\sigma-1}$

$$
-\lambda M f_{e} \frac{h(x)}{1-G(x)}+\zeta M m_{\exp } h(x) a \alpha^{\rho} \theta y^{\sigma-1}=0,
$$

$(y): \quad \partial £ / \partial y=\lambda M f_{\exp } m_{\exp } h(y)+\lambda M m_{\exp } h(y) \alpha \theta y^{\sigma-1}-\lambda M m_{\exp } \alpha \theta(\sigma-1) y^{\sigma-2}$

$$
-\zeta M m_{\exp } h(y) a \alpha^{\rho} \theta y^{\sigma-1}+\zeta M m_{\exp } a \alpha^{\rho} \theta(\sigma-1) y^{\sigma-2}=0,
$$

$(\phi): \quad \partial £ / \partial \phi=M \rho \phi^{\rho-1} \theta x^{\sigma-1}-\lambda M \theta x^{\sigma-1}=0$,

$(\alpha): \quad \partial £ / \partial \alpha=-\lambda M m_{\exp } \theta y^{\sigma-1}+\zeta M m_{\exp } a \rho \alpha^{\rho-1} \theta y^{\sigma-1}=0$,

$(M): \quad \partial £ / \partial M=\phi^{\rho} \theta x^{\sigma-1}-\lambda f-\lambda \theta \phi x^{\sigma-1}-\lambda f_{\exp } m_{\exp }-\lambda m_{\exp } \alpha \theta y^{\sigma-1}-\lambda \frac{f_{e}}{1-G(x)}+\zeta m_{\exp } a \alpha^{\rho} \theta y^{\sigma-1}=0$.

Note that $\theta(\sigma-1) v^{\sigma-2}=(\theta-1) h(v) v^{\sigma-1}$, hence, we have 8 equations with 8 unknown variables:

$$
\begin{aligned}
& (z): \frac{\beta-\rho}{\beta} z^{\rho}\left(\frac{(\sigma-1) F_{\exp }}{\gamma}\right)^{\rho}=\zeta \sigma F_{\exp }, \\
& (x): \quad \phi(\theta-1) x^{\sigma-1}\left(\phi^{\rho-1}-\lambda\right)-\lambda m_{\exp } f_{\exp }-\lambda m_{\exp } \theta \alpha y^{\sigma-1}-\lambda f_{e} \frac{1}{1-G(x)}+\zeta m_{\exp } a \alpha^{\rho} \theta y^{\sigma-1}=0, \\
& \text { (y) }: \quad \lambda f_{\exp }+\lambda \alpha \theta y^{\sigma-1}-\lambda \alpha(\theta-1) y^{\sigma-1}-\zeta a \alpha^{\rho} \theta y^{\sigma-1}+\zeta a \alpha^{\rho}(\theta-1) y^{\sigma-1} \\
& =\lambda f_{\exp }+\lambda \alpha y^{\sigma-1}-\zeta a \alpha^{\rho} y^{\sigma-1}=0, \\
& (\phi) \quad: \quad \rho \phi^{\rho-1}=\lambda, \\
& (\alpha) \quad: \quad \zeta a \rho \alpha^{\rho-1}=\lambda, \\
& (M): \quad \phi^{\rho} \theta x^{\sigma-1}-\lambda f-\lambda \theta \phi x^{\sigma-1}-\lambda f_{\exp } m_{\exp }-\lambda m_{\exp } \alpha \theta y^{\sigma-1}-\lambda \frac{f_{e}}{1-G(x)}+\zeta m_{\exp } a \alpha^{\rho} \theta y^{\sigma-1}=0, \\
& (F E) \quad: \quad 1=M f+M \theta \phi x^{\sigma-1}+M f_{\exp } m_{\exp }+M m_{\exp } \alpha \theta y^{\sigma-1}+\frac{M f_{e}}{1-G(x)}, \\
& (T B) \quad: \quad \sigma F_{\exp } b^{\beta} z^{-\beta}=M m_{\exp } a \alpha^{\rho} y^{\sigma-1} .
\end{aligned}
$$


Moreover, subtracting (M) from (x) gives:

$$
-\phi^{\rho} x^{\sigma-1}+\lambda \phi x^{\sigma-1}+\lambda f=0, \quad \text { or } \quad \lambda \phi x^{\sigma-1}-\phi^{\rho} x^{\sigma-1}=\lambda f .
$$

From $(\phi), \lambda=\rho \phi^{\rho-1}$. Thus, $(x)$ is $\phi x^{\sigma-1}=\sigma \rho f$. Similarly, using $(\alpha)$ in (y) gives $\alpha y^{\sigma-1}=\sigma \rho f_{\exp }$. Using new equations $(\mathrm{x}),(\mathrm{y}),(\phi)$, and $(\alpha)$, we derive new $(\mathrm{M})$ and $(\mathrm{FE})$ conditions:

$$
\begin{gathered}
\frac{f_{e}}{1-G(x)}=\theta(\sigma \rho f)\left(\frac{1}{\rho}-1\right)-f-f_{\exp } m_{\exp }+\theta m_{\exp }\left(\sigma \rho f_{\exp }\right)\left(\frac{1}{\rho}-1\right)=(\theta-1)\left(f+f m_{\exp }\right), \\
1=M\left[f+\theta \sigma \rho f+f_{\exp } m_{\exp }+m_{\exp } \theta \sigma \rho f_{\exp }+(\theta-1)\left(f+f m_{\exp }\right)\right]=\sigma \theta M\left(f+m_{\exp } f_{\exp }\right) .
\end{gathered}
$$

Thus, we have the following system of F.O.C.s in the social planner's problem:

$$
\begin{array}{rll}
(z) & : \quad \frac{\beta-\rho}{\beta} z^{\rho}\left(\frac{(\sigma-1) F_{\exp }}{\gamma}\right)^{\rho}=\zeta \sigma F_{\exp }, \\
(x) & : \quad \phi x^{\sigma-1}=\sigma \rho f, \\
(y) & : \quad \alpha y^{\sigma-1}=\sigma \rho f_{\exp }, \\
(\phi) \quad: \quad \rho \phi^{\rho-1}=\lambda, \\
(\alpha) \quad: \quad \zeta a \rho \alpha^{\rho-1}=\lambda, \\
(M) \quad: \quad \frac{f_{e}}{1-G(x)}=(\theta-1)\left(f+m_{\exp } f_{\exp }\right), \\
(F E) \quad: \quad 1=\sigma \theta M\left(f+m_{\exp } f_{\exp }\right), \\
(T B) \quad: \quad \sigma F_{\exp } b^{\beta} z^{-\beta}=M m_{\exp } a \alpha^{\rho} y^{\sigma-1} .
\end{array}
$$

Uniqueness of the Solution It can be shown that if there are 2 solutions, and both solutions have at least one common component (for example, $x_{1}=x_{2}$ ), then these solutions coincide. We will prove that there should be a unique $x$, which solves the system, thus, if the solution exists, it is unique. To do this, we will rewrite the system above till we have 1 equation with 1 unknown variable, which has a unique solution. First, let us exclude $M$. From (TB), $M=$ $\sigma F_{\exp } b^{\beta} z^{-\beta} /\left(m_{\exp } a \alpha^{\rho} \theta y^{\sigma-1}\right) . M$ is used only in (FE), which together with $(y)$ and $(\alpha)$ can be written as:

$$
\begin{aligned}
(F E): & 1=\sigma \theta \frac{\sigma F_{\exp } b^{\beta} z^{-\beta}\left(f+m_{\exp } f_{\exp }\right)}{m_{\exp } a \alpha^{\rho} y^{\sigma-1}}=\sigma \theta \frac{F_{\exp } b^{\beta} z^{-\beta}\left(f+m_{\exp } f_{\exp }\right)}{m_{\exp } a \alpha^{\rho-1} \rho f_{\exp }} \\
= & \sigma \theta \frac{F_{\exp } b^{\beta} z^{-\beta}\left(f+m_{\exp } f_{\exp }\right)}{m_{\exp } f_{\exp } \lambda / \zeta}=\frac{\zeta}{\lambda} \sigma \theta \frac{F_{\exp } b^{\beta} z^{-\beta}\left(f+m_{\exp } f_{\exp }\right)}{m_{\exp } f_{\exp }} .
\end{aligned}
$$


Let us exclude $\lambda$ and $\zeta$. From $(z)$ and $(\phi): \zeta=\frac{1}{\sigma F_{\exp }} \frac{\beta-\rho}{\beta} z^{\rho}\left(\frac{(\sigma-1) F_{\exp }}{\gamma}\right)^{\rho}$ and $\lambda=\rho \phi^{\rho-1}$. Then,

$$
\begin{aligned}
(x) \quad: \quad \phi x^{\sigma-1}=(\sigma-1) f ; \quad(y): \quad \alpha y^{\sigma-1}=(\sigma-1) f_{\exp } ; \\
(\alpha) \quad: \quad z^{\rho-1} a \rho \alpha^{\rho-1}=\phi^{\rho-1} ; \quad(M): \quad \frac{f_{e}}{1-G(x)}=(\theta-1)\left(f+m_{\exp } f_{\exp }\right) ; \\
(F E) \quad: \quad 1=\frac{\beta-\rho}{\rho \beta}\left(\frac{z(\sigma-1) F_{\exp }}{\gamma}\right)^{\rho} \frac{1}{\phi^{\rho-1}} \theta\left(\frac{b}{z}\right)^{\beta} \frac{\left(f+m_{\exp } f_{\exp }\right)}{m_{\exp } f_{\exp }} .
\end{aligned}
$$

Let us exclude $z$. From $(\alpha),(x)$, and $(y), z=(a \rho)^{\frac{1}{\rho-1}} \frac{\phi}{\alpha}=(a \rho)^{\frac{1}{\rho-1}} \frac{f}{f_{\exp }}\left(\frac{y}{x}\right)^{\sigma-1}$, so we have now

$$
\begin{aligned}
(x): & \phi x^{\sigma-1}=(\sigma-1) f, \quad(y): \quad \alpha y^{\sigma-1}=(\sigma-1) f_{\exp }, \\
(M) & : \quad \frac{f_{e}}{1-G(x)}=(\theta-1)\left(f+m_{\exp } f_{\exp }\right), \\
(F E) & : \quad \text { Some constant }=\alpha^{1-\rho}\left(m_{\exp }\right)^{\frac{(\sigma-1)(1-\beta)}{\beta}} \frac{f+m_{\exp } f_{\exp }}{f_{\exp } m_{x}} .
\end{aligned}
$$

Let us exclude $\phi$ and $\alpha$. From $(x)$ and $(y): \phi=\frac{(\sigma-1) f}{x^{\sigma-1}} \quad$ and $\quad \alpha=\frac{(\sigma-1) f_{\exp }}{y^{\sigma-1}}$. Thus,

$$
\begin{aligned}
(M) & : \quad x=\left(b^{\beta} \frac{(\theta-1)}{f_{e}}\left(f+m_{\exp } f_{\exp }\right)\right)^{1 / \beta}, \\
(F E) & : \quad \text { Some constant }=y^{-\rho}\left(m_{\exp }\right)^{\frac{(\sigma-1)(1-\beta)}{\beta}} \frac{f+m_{\exp } f_{\exp }}{f_{\exp } m_{x}}, \text { or } \\
(F E): & \text { Some constant }=\left(m_{\exp }\right)^{\frac{(\sigma-1)(1-\beta)}{\beta}}\left[\frac{f+m_{\exp } f_{\exp }}{f_{\exp } m_{x}}\right]^{1-\frac{\rho}{\beta}} .
\end{aligned}
$$

Note that equation (FE) can be rewritten as

$$
\left(m_{\exp }\right)^{\frac{(\sigma-1)(1-\beta)}{\beta}}\left(\frac{f}{m_{\exp }}+f_{\exp }\right)^{1-\frac{\rho}{\beta}}=\text { Some exogenously given constant. }
$$

Then since $\beta>\sigma>1>\rho$, the left-hand side of equation above is a decreasing function of $m_{\text {exp }}$. Thus, the equation above has a unique solution $m_{\exp }$. But from (M), it follows, that $x$ is also unique! Therefore, we proved that if the solution of the system of F.O.C.s exists, it is unique.

Sufficiency of F.O.C.s Let us rewrite the Lagrangian as

$$
£=U+H, \text { where } U=\left(\frac{(\sigma-1) F_{\text {exp }}}{\gamma}\right)^{\rho} b^{\beta} z^{\rho-\beta}+M \phi^{\rho} \theta x^{\sigma-1} \text { and } H=(-\lambda, \zeta) \vec{h},
$$


where $\vec{h}$ is a vector of restrictions in our problem:

$$
\vec{h}=\left(\begin{array}{c}
M f+M \theta \phi x^{\sigma-1}+M m_{\exp } f_{\exp }+M m_{\exp } \theta \alpha y^{\sigma-1}+\frac{M f_{e}}{b^{\beta}} x^{\beta}-1 \\
M m_{\exp } a \alpha^{\rho} y^{\sigma-1}-\sigma F_{\exp } b^{\beta} z^{-\beta}
\end{array}\right) .
$$

To prove the sufficiency of the first order conditions of the social planner's problem described above, we need to show that for any vector $\vec{\chi}$ such that ${ }^{24}$

$$
\vec{\chi} \neq \overrightarrow{0} \quad \text { and } \quad \nabla \vec{h}(\text { solution }) \vec{\chi}=\overrightarrow{0},
$$

we have

$$
\vec{\chi}^{\prime} £_{\xi \xi}\left(\xi^{*}\right) \vec{\chi}<0,
$$

where $£_{\xi \xi}\left(\xi^{*}\right)$ is the matrix of second derivatives of the Lagrangian with respect to $\xi^{\prime}=(x, y, z, M, \alpha, \phi)$, evaluated at the solution point $\xi^{*}$. (See, for example, Theorem 3.3.2, p. 214 in Giorgi, Guerraggio Thierfelder (2004), which states that if there exist such $\lambda$ and $\zeta$, for which the conditions above are satisfied, then the solution we found is a point of global maximum of the objective function subject to our restrictions. And we found such $\lambda$ and $\zeta$ already.) In order to do this, we can show that the matrix of the second derivatives can be written as (see the proof below):

$$
£_{\xi \xi}\left(\xi^{*}\right)=\left(\begin{array}{cccccc}
a_{11} & 0 & 0 & 0 & 0 & 0 \\
0 & a_{22} & 0 & 0 & 0 & 0 \\
0 & 0 & a_{33} & 0 & 0 & 0 \\
0 & 0 & 0 & a_{44} & 0 & 0 \\
0 & 0 & 0 & 0 & a_{55} & 0 \\
0 & 0 & 0 & 0 & 0 & 0
\end{array}\right),
$$

where $a_{i i}<0$ for any $i \neq 6$. Thus, $\vec{\chi}^{\prime} £_{\xi \xi}\left(\xi^{*}\right) \vec{\chi}=\sum_{i=1}^{5} a_{i i} \chi_{i}^{2} \leq 0$, so we need to show that $\vec{\chi}^{\prime} £_{\xi \xi}\left(\xi^{*}\right) \vec{\chi} \neq 0$. Note that the only way $\vec{\chi}^{\prime} £_{\xi \xi}\left(\xi^{*}\right) \vec{\chi}=0$ is if $\vec{\chi}^{\prime}=\left(0,0,0,0,0, \chi_{6}\right)$ and $\chi_{6} \neq 0$. However, in this case (37) is violated since $\nabla \vec{h}$ (solution) $\vec{\chi}=\overrightarrow{0}$ implies

$$
\begin{gathered}
{\left[\frac{\partial}{\partial M}\left(M f+M \theta \phi x^{\sigma-1}+M m_{\exp } f_{\exp }+M m_{\exp } \theta \alpha y^{\sigma-1}+\frac{M f_{e}}{b^{\beta}} x^{\beta}-1\right)\right] \chi_{6}=0,} \\
{\left[\frac{\partial}{\partial M}\left(M m_{\exp } a \alpha^{\rho} y^{\sigma-1}-\sigma F_{\exp } b^{\beta} z^{-\beta}\right)\right] \chi_{6}=0, \quad \text { or }} \\
\left(f+\theta \phi x^{\sigma-1}+m_{\exp } f_{\exp }+m_{\exp } \theta \alpha y^{\sigma-1}+\frac{f_{e}}{b^{\beta}} x^{\beta}\right) \chi_{6}=0, \quad \text { and } \quad\left(m_{\exp } a \alpha^{\rho} y^{\sigma-1}\right) \chi_{6}=0,
\end{gathered}
$$

which is clearly impossible, since in the second equation above, $m_{\exp } a \alpha^{\rho} \theta y^{\sigma-1} \neq 0$ and $\chi_{6} \neq 0$.

The derivation of $£_{\xi \xi}\left(\xi^{*}\right)$. Let denote the elements of $£_{\xi \xi}\left(\xi^{*}\right)$ by $\left[a_{i j}\right]_{i, j=1, . ., 6}$.

\footnotetext{
${ }^{24}$ In the expression below, $\nabla \vec{h}$ (solution) is a matrix of the first derivatives of the vector of restrictions in our problem with respect to $\xi^{\prime}=(z, x, y, \phi, \alpha, M)$, evaluated at the solution point $\xi^{*}$.
} 
Diagonal elements. First, note that

$$
\begin{aligned}
a_{11} & =\frac{\partial^{2} £}{\partial z^{2}}=(\rho-\beta)(\rho-\beta-1)\left(\frac{(\sigma-1) F_{\exp }}{\gamma}\right)^{\rho} b^{\beta} z^{\rho-\beta-2}-\zeta \beta(\beta+1) \sigma F_{\exp } b^{\beta} z^{-\beta-2} \\
& =\zeta \sigma F_{\exp } b^{\beta} z^{-\beta-2} \beta(-\rho)<0,
\end{aligned}
$$

where the second equality follows from equation $(z)$.

What we do in cases $i=2,3$ is we take the derivatives and use the property that $\frac{\partial\left(x^{n}\right)}{\partial x}=$ $\frac{n}{x}\left(x^{n}\right)$. Then we use the corresponding condition to simplify the expression for the derivative and compare it with 0. For example, since $\partial\left(m_{\exp } h(x)\right) / \partial x=\partial\left(\beta x^{\beta-1} / y^{\beta}\right) / \partial x=\frac{\beta-1}{x} m_{\exp } h(x)$ and $\partial\left(\frac{h(x)}{1-G(x)}\right) / \partial x=\partial\left(\beta x^{\beta-1}\right) / \partial x=\frac{\beta-1}{x} \frac{h(x)}{1-G(x)}$,

$$
\begin{aligned}
a_{22}= & \frac{\partial^{2} £}{\partial x^{2}}=M \phi^{\rho} \theta(\sigma-1)(\sigma-2) x^{\sigma-3}-\lambda \phi \theta(\sigma-1)(\sigma-2) x^{\sigma-3} \\
& -\frac{\beta-1}{x}\left(\lambda M m_{\exp } f_{\exp } h(x)-\lambda M m_{\exp } h(x) \theta \alpha y^{\sigma-1} \lambda M f_{e} \frac{h(x)}{1-G(x)}+\zeta M m_{\exp } h(x) a \alpha^{\rho} \theta y^{\sigma-1}\right) .
\end{aligned}
$$

We can use condition $(\mathrm{x})$ to rewrite it as

$$
\begin{gathered}
a_{22}=M \phi^{\rho} \theta(\sigma-1)(\sigma-2) x^{\sigma-3}-\lambda \phi \theta(\sigma-1)(\sigma-2) x^{\sigma-3}-\frac{\beta-1}{x}\left[\phi^{\rho} \theta(\sigma-1) x^{\sigma-2}-\lambda \phi \theta(\sigma-1) x^{\sigma-2}\right] \\
=M \phi \theta(\sigma-1) x^{\sigma-3}\left[\phi^{\rho-1}-\lambda\right][(\sigma-1)-\beta] .
\end{gathered}
$$

Note that since $\rho \phi^{\rho-1}=\lambda$ and $\rho<1$, then $\phi^{\rho-1}-\lambda>0$, while $(\sigma-1)-\beta<0$. Thus, $a_{22}<0$. Similarly, it can be shown that $a_{33}=\frac{\partial^{2} £}{\partial y^{2}}=-(\sigma-1) M \lambda f_{\exp } m_{\exp } h(y) \frac{1}{y}<0, a_{44}=$ $\frac{\partial^{2} £}{\partial \phi^{2}}=(\rho-1) \phi^{\rho-2} M \rho \theta x^{\sigma-1}<0$, and $a_{55}=\frac{\partial^{2} £}{\partial \alpha^{2}}=(\rho-1) \zeta M m_{\exp } a \rho \alpha^{\rho-2} \theta y^{\sigma-1}<0$, while $a_{66}=\frac{\partial^{2} £}{\partial M^{2}}=\frac{\partial}{\partial M}\left(\frac{\partial £}{\partial M}\right)=0$.

Off-Diagonal elements. To derive the off diagonal elements, we use Young's theorem. As a result,

$$
\begin{gathered}
a_{12}=a_{21}=\frac{\partial^{2} £}{\partial z \partial x}=\frac{\partial}{\partial x}\left(\frac{\partial £}{\partial z}\right)=\frac{\partial}{\partial x}\left(\rho z^{\rho-1}-\zeta\right)=0, \text { and similarly, } \\
a_{13}=a_{31}=a_{14}=a_{41}=a_{15}=a_{51}=a_{16}=a_{61}=0 . \\
a_{23}=a_{32}=\frac{\partial}{\partial x}\left(\frac{\partial £}{\partial y}\right)=\frac{\beta}{x}\left(\frac{\partial £}{\partial y}\right)=0, \text { as } \frac{\partial m_{\exp }}{\partial x}=\frac{\beta}{x} m_{\exp } \\
a_{24}=a_{42}=\frac{\partial}{\partial x}\left(\frac{\partial £}{\partial \phi}\right)=\frac{\sigma-1}{x}\left(\frac{\partial £}{\partial \phi}\right)=0, a_{25}=a_{52}=\frac{\partial}{\partial x}\left(\frac{\partial £}{\partial \alpha}\right)=\frac{\beta}{x}\left(\frac{\partial £}{\partial \alpha}\right)=0 \\
a_{26}=a_{62}=\frac{\partial^{2} £}{\partial M \partial x}=\frac{\partial}{\partial M}\left(\frac{\partial £}{\partial x}\right)=\frac{1}{M}\left(\frac{\partial £}{\partial x}\right)=0 .
\end{gathered}
$$

Using the same logic, it can be shown that all off-diagonal elements of the matrix are zeros. 


\subsubsection{The Consumption Subsidy}

The Optimal Value of Consumption Subsidy. We need to rewrite the equilibrium conditions derived in Section 2 by setting $s=1$ :

$$
\begin{gathered}
\text { (EXP) condition } A\left(\frac{\rho y}{w}\right)^{\sigma-1}=\sigma w f_{\exp }, \\
\text { (FE) condition } \quad(\theta-1) x^{-\beta}\left[f+m_{\exp } f_{\exp }\right]=\frac{f_{e}}{b^{\beta}}, \\
(\mathrm{TB}) \text { condition } M_{m} F_{\exp }=w M_{\exp } f_{\exp }, \\
(\mathrm{M}) \text { condition } M=\frac{1}{\sigma \theta\left(f+m_{\exp } f_{\exp }\right)}=\frac{(\theta-1) b^{\beta}}{\sigma \theta f_{e}} x^{-\beta} . \\
\text { (z) condition } 1=\sigma \theta M\left[f_{\exp } m_{\exp }+(1-\eta) f+\frac{F_{\exp }}{w}\left(\frac{\gamma}{\eta} \frac{x}{w z}\right)^{\sigma-1}\right]
\end{gathered}
$$

Now we are ready to prove that a consumption subsidy equal to $1-\eta$, where $\eta=\frac{\beta-\rho}{\beta}$, results in the maximal level of welfare.

Proof. Step 1. First, we prove that when $\eta$ rises, $y$ and $w$ must fall and $x$ must rise. From

$$
(\mathrm{EXP}): w^{\sigma}=\frac{A \rho^{\sigma-1}}{\sigma f_{\exp }} y^{\sigma-1}
$$

$y$ and $w$ must move in the same direction, and from the (FE) condition, $x$ and $y$ move in the opposite direction. Next,

$$
\begin{gathered}
(\mathrm{z})+(\mathrm{M}) \Rightarrow 1=\frac{1}{\left(f+m_{\exp } f_{\exp }\right)}\left[f_{\exp } m_{\exp }+(1-\eta) f+\frac{F_{\exp }}{w}\left(\frac{\gamma}{\eta} \frac{x}{w z}\right)^{\sigma-1}\right], \text { or } \\
\frac{F_{\exp }}{w}\left(\frac{\gamma}{\eta} \frac{x}{w z}\right)^{\sigma-1}=\eta f, \text { or } \\
w^{\sigma}=\frac{1}{\eta^{\sigma}} \frac{F_{\exp }}{f}\left(\frac{\gamma x}{z}\right)^{\sigma-1},
\end{gathered}
$$

where from (TB) condition, $z^{-1}=b^{-1}\left(\frac{f_{\exp }}{F_{\exp }} w M_{\exp }\right)^{\frac{1}{\beta}} \propto w^{\frac{1}{\beta}} y^{-1}$ so that

$$
w^{\sigma-\frac{\sigma-1}{\beta}} \propto \frac{1}{\eta^{\sigma}}\left(\frac{x}{y}\right)^{\sigma-1} .
$$

Now, assume that if $\eta$ rises, then $y$ rises and $x$ falls. Then from (43), $w \propto y^{\rho}$ must rise, but

$$
\text { from (46): } \left.w \propto\left[\frac{1}{\eta^{\sigma}}\left(\frac{x}{y}\right)^{\sigma-1}\right]^{\frac{1}{\sigma-\frac{\sigma-1}{\beta}}} \text { must fall (since } \beta>\sigma\right) \text {. }
$$


Our assumption led to the contradiction, thus, as $\eta$ rises, $y$ and $w$ must fall and $x$ must increase.

Step 2. Note that from (z) condition,

$$
\begin{gathered}
\int_{z}^{\infty}\left[q_{m}(\varphi)\right]^{\rho} M_{m} \mu(\varphi) d \varphi=M_{m}\left[\frac{F_{\exp }}{\gamma}(\sigma-1)\right]^{\rho} z^{\beta-\rho(\sigma-1)} \beta \int_{z}^{\infty} \varphi^{\rho \sigma-\beta-1} d \varphi=M_{m} \theta\left[\frac{F_{\exp }}{\gamma}(\sigma-1) z\right]^{\rho}, \\
\quad \text { and } \int_{x}^{\infty}[q(\varphi)]^{\rho} M \mu(\varphi) d \varphi=M[f(\sigma-1)]^{\rho} x^{\beta-\rho(\sigma-1)} \beta \int_{x}^{\infty} \varphi^{\rho \sigma-\beta-1} d \varphi=M \theta[f(\sigma-1) x]^{\rho} .
\end{gathered}
$$

We want to rewrite

$$
U^{\rho}=\int_{z}^{\infty}\left[q_{m}(\varphi)\right]^{\rho} M_{m} \mu(\varphi) d \varphi+\int_{x}^{\infty} q(\varphi)^{\rho} M \mu(\varphi) d \varphi=\theta(\sigma-1)^{\rho}\left[M_{m}\left(\frac{F_{\exp }}{\gamma} z\right)^{\rho}+M(f x)^{\rho}\right]
$$

as $U^{\rho}=d(\eta) h(\eta)$, where $d(\eta) \equiv \theta(\sigma-1)^{\rho} M(f x)^{\rho}$ falls faster than $h(\eta) \equiv \frac{M_{m}}{M}\left(\frac{F_{\exp } z}{f \gamma x}\right)^{\rho}+1$ rises, if $\eta>\frac{\beta-\rho}{\beta}$, and vice verse if $\eta<\frac{\beta-\rho}{\beta}$. In other words, the utility is maximal at $\eta=\frac{\beta-\rho}{\beta}$ !

First, note that $x$ rises as $\eta$ rises, so we can rewrite both functions and look at their behavior as functions of $x$. Then we can compare the elasticities of these two functions and show that $\varepsilon_{d}<0<\varepsilon_{h}$. Moreover, if $\eta>\frac{\beta-\rho}{\beta}\left(\eta<\frac{\beta-\rho}{\beta}\right)$, then $\left|\varepsilon_{d}\right|>\left|\varepsilon_{h}\right|\left(\left|\varepsilon_{d}\right|<\left|\varepsilon_{h}\right|\right)$, so that $d(x)$ falls faster (slower) than $h(x)$ rises, and $U=d(x) h(x)$ falls (rises) as a result.

First, since $\rho<1<\beta, d(x)$ is decreasing in $x$ :

$$
d(x)=\theta(\sigma-1)^{\rho} M(f x)^{\rho}=\theta(\sigma-1)^{\rho} f^{\rho}\left[\frac{(\theta-1) b^{\beta}}{\sigma \theta f_{e}}\right](x)^{\rho-\beta}, \text { and } \varepsilon_{d}=\rho-\beta<0 .
$$

Second, $h(x)=\frac{M_{m}}{M}\left(\frac{F_{\exp } z}{f \gamma x}\right)^{\rho}+1$. Note that from the (TB) condition,

$$
h(x)=w m_{x} \frac{f_{\exp }}{F_{\exp }}\left(\frac{F_{\exp } z}{f \gamma x}\right)^{\rho}+1 .
$$

Thus, $h(x)=1+\kappa(x)$, where from (43),

$$
\kappa(x)=w m_{x} \frac{f_{\exp }}{F_{\exp }}\left(\frac{F_{\exp } z}{f \gamma x}\right)^{\rho} \propto y^{\rho}\left(\frac{x}{y}\right)^{\beta}\left(\frac{z}{x}\right)^{\rho} .
$$

Moreover, since $z^{-1} \propto w^{\frac{1}{\beta}} y^{-1}=y^{\frac{\rho}{\beta}-1}$, then $\kappa(x) \propto\left(\frac{x}{y}\right)^{\beta-\rho}\left(y^{1-\frac{\rho}{\beta}}\right)^{\rho}=x^{\beta-\rho} y^{(\beta-\rho)\left(\frac{\rho}{\beta}-1\right)}$. Since $\beta>1>\rho$ and $y$ falls as $x$ rises, then $\kappa(x)$, and in turn $h(x)$, is increasing with $x$.

$$
\varepsilon_{h}=\frac{h^{\prime}(x)}{h(x)} x=\frac{\kappa^{\prime}(x)}{1+\kappa(x)} x=\frac{\kappa^{\prime}(x)}{\kappa(x)} x \frac{\kappa(x)}{1+\kappa(x)}=\varepsilon_{\kappa} \frac{\kappa(x)}{1+\kappa(x)} .
$$


To calculate $\varepsilon_{\kappa}$, we use two properties: $\varepsilon_{a(x) b(x)}=\varepsilon_{a(x)}+\varepsilon_{b(x)}$ and $\varepsilon_{a(b(x))}=\varepsilon_{a(b)} \varepsilon_{b(x)}$. Then,

$$
\varepsilon_{\kappa(x)}=\varepsilon_{x^{\beta-\rho}}+\varepsilon_{y^{(\beta-\rho)}\left(\frac{\rho}{\beta}-1\right)}=(\beta-\rho)+(\beta-\rho)\left(\frac{\rho}{\beta}-1\right) \varepsilon_{y(x)} .
$$

From the (FE) condition, $\varepsilon_{y(x)}=-\frac{f}{f_{\exp }}\left(\frac{y}{x}\right)^{\beta}$, so that $\varepsilon_{\kappa(x)}=(\beta-\rho)\left(1+\left(1-\frac{\rho}{\beta}\right) \frac{f}{f_{\exp }}\left(\frac{y}{x}\right)^{\beta}\right)$,

$$
\varepsilon_{h}=(\beta-\rho)\left(1+\left(1-\frac{\rho}{\beta}\right) \frac{f}{f_{\exp }}\left(\frac{y}{x}\right)^{\beta}\right) \frac{\kappa(x)}{\gamma+\kappa(x)}>0 .
$$

Finally, we can compare the absolute values of elasticities from (47) and (48):

$$
\begin{gathered}
\left|\varepsilon_{d}\right|=\beta-\rho \text { versus }\left|\varepsilon_{h}\right|=(\beta-\rho)\left(1+\left(1-\frac{\rho}{\beta}\right) \frac{f}{f_{\exp }}\left(\frac{y}{x}\right)^{\beta}\right) \frac{\kappa(x)}{1+\kappa(x)} \text {, or } \\
1 \text { versus }\left(1+\left(1-\frac{\rho}{\beta}\right) \frac{f}{f_{\exp }}\left(\frac{y}{x}\right)^{\beta}\right) \frac{\kappa(x)}{1+\kappa(x)} \text {, or } \frac{1}{\kappa(x)} \text { versus }\left(1-\frac{\rho}{\beta}\right) \frac{f}{f_{\exp }}\left(\frac{y}{x}\right)^{\beta} \text {, or } \\
\frac{\beta}{\beta-\rho} \frac{f_{\exp }}{f}\left(\frac{x}{y}\right)^{\beta} \frac{1}{\kappa(x)} \text { versus } 1 .
\end{gathered}
$$

To compare the left-hand side with 1 , we plug the expressions for $\kappa(x)$ and use (44):

$$
\begin{aligned}
\frac{\beta}{\beta-\rho} \frac{\frac{f_{\exp }}{f}\left(\frac{x}{y}\right)^{\beta}}{w m_{x} \frac{f_{\exp }}{F_{\exp }}\left(\frac{F_{\exp } z}{f \gamma x}\right)^{\rho}} & =\frac{\beta}{\beta-\rho} \frac{\frac{F_{\exp }}{f}}{w\left(\frac{F_{\exp } z}{f \gamma x}\right)^{\rho}}=\frac{\beta}{\beta-\rho}\left(\frac{F_{\exp }}{f}\right)^{\frac{1}{\sigma}}\left(\frac{\gamma x}{z}\right)^{\rho} \frac{1}{w} \\
& =\frac{\beta}{\beta-\rho}\left(\frac{F_{\exp }}{f}\right)^{\frac{1}{\sigma}}\left(\frac{\gamma x}{z}\right)^{\rho} \frac{1}{\frac{1}{\eta}\left(\frac{F_{\exp }}{f}\right)^{\frac{1}{\sigma}}\left(\frac{\gamma x}{z}\right)^{\rho}}=\frac{\beta}{\beta-\rho} \eta .
\end{aligned}
$$

Thus, the comparison in (49) results in comparing $\eta$ with $\frac{\beta-\rho}{\beta}$, and we proved our results.

First Best Allocation and Consumption Subsidy. As shown before, the market equilibrium with a consumption subsidy $\eta=\frac{\beta-\rho}{\beta}$ satisfies:

$$
\begin{gathered}
(M 1): R P^{\sigma-1} \eta^{-\sigma} w^{-\sigma}(\rho x)^{\sigma-1}=\sigma f, \quad(M 2): A w^{-\sigma}(\rho y)^{\sigma-1}=\sigma f_{\exp }, \\
(M 3): \int_{z}^{\infty} r_{m}(\varphi) M_{m} \mu(\varphi) d \varphi=M_{m} \theta\left[\frac{F_{\exp }}{\gamma}(\sigma-1)\right], \\
(M 4): \frac{f_{e}}{1-G(x)}=\left(f+m_{\exp } f_{\exp }\right)(\theta-1), \quad(M 5): M \sigma \theta\left(f+m_{\exp } f_{\exp }\right)=1,
\end{gathered}
$$

where $(P / w)^{1-\sigma}=\theta M_{m}\left(\frac{\rho z}{w \gamma}\right)^{\sigma-1}+\theta M\left(\frac{\rho x}{\eta}\right)^{\sigma-1}$, i.e., we have 5 equations with 5 unknown variables, $R, w, x, y, M$. If we have a market equilibrium and an optimal allocation $\left(z_{0}, x_{0}, y_{0}, \alpha, \phi, M_{0}, \lambda, \zeta\right)$, which satisfies the system of equations (29)-(36), is it the case that $\left(x_{0}, y_{0}, z_{0}, M_{0}\right)=\left(x_{M}, y_{M}, z_{M}, M_{M}\right)$ ? 
The first indication that this is the case is that equations (M) and (FE) in the optimal allocation coincide with equations (M4) and (M5) in the market equilibrium.

One way to complete the answer (assuming the solutions are unique) is to postulate $\left(x_{0}, y_{0}, z_{0}, M_{0}\right)=$ $\left(x_{M}, y_{M}, z_{M}, M_{M}\right)$ and then see if there exist $(\alpha, \phi, \lambda, \zeta)$ such that these together with $\left(x_{M}, y_{M}, z_{M}, M_{M}\right)$ satisfy 8 equations for an optimum allocation. This is exactly the case if:

$$
\begin{aligned}
& \phi=R P^{\sigma-1} w^{-\sigma} \rho^{\sigma}, \quad \alpha=A w^{-\sigma} \rho^{\sigma}, \quad \zeta=\frac{\beta-\rho}{\beta} R^{\rho-1} P^{-\rho}, \quad \lambda=R^{\rho-1} P^{-\rho} \frac{\beta-\rho}{\beta} w, \text { so that } \\
& (z): \quad \rho \frac{\beta-\rho}{\beta} z^{\rho}\left(\frac{(\sigma-1) F_{\exp }}{\gamma}\right)^{\rho}=\frac{\beta-\rho}{\beta} R^{\rho-1} P^{-\rho} \sigma F_{\exp } \text { or } R P^{\sigma-1}\left(\frac{\gamma}{\rho z}\right)^{1-\sigma}=\sigma F_{\exp },
\end{aligned}
$$

(formula for import demand in $\mathrm{ME}$ ),

$(x): \quad R P^{\sigma-1} w^{-\sigma}\left(\frac{\beta-\rho}{\beta}\right)^{-\sigma} \rho^{\sigma} x^{\sigma-1}=\sigma \rho f$ or $R P^{\sigma-1} \eta^{-\sigma}\left(\frac{\rho x}{w}\right)^{\sigma-1}=\sigma w f$,

(zero profit condition for domestic producers in ME, M1)

$$
\text { (y) : } A w^{-\sigma} \rho^{\sigma} y^{\sigma-1}=\sigma \rho f_{\exp } \text { or } A w^{1-\sigma}(\rho y)^{\sigma-1}=\sigma w f_{\exp },
$$

(zero profit condition for exporters in ME, M2)

$(\phi): \quad \rho\left[R P^{\sigma-1} w^{-\sigma}\left(\frac{\beta-\rho}{\beta}\right)^{-\sigma} \rho^{\sigma}\right]^{\rho-1}=R^{\rho-1} P^{-\rho} \frac{\beta-\rho}{\beta} w, \quad$ is an identity.

$(\alpha): \frac{\beta-\rho}{\beta} R^{\rho-1} P^{-\rho} A^{1 / \sigma} \rho\left(A w^{-\sigma} \rho^{\sigma}\right)^{\rho-1}=R^{\rho-1} P^{-\rho} \frac{\beta-\rho}{\beta} w$ is an identity.

$(M): \frac{f_{e}}{1-G(x)}=(\theta-1)\left(f+m_{\exp } f_{\exp }\right), \quad$ (free entry condition in ME, M4),

$(F E): \quad 1=\sigma \theta M\left(f+m_{\exp } f_{\exp }\right)$ or $M=\frac{1}{\sigma \theta\left(f+f_{\exp } m_{\exp }\right)}$,

(the expression for the mass of active firms in ME, M5)

$(T B): \quad \sigma F_{\exp } b^{\beta} z^{-\beta}=M m_{\exp } a \alpha^{\rho} y^{\sigma-1}$ or $\quad M_{m} F_{\exp }=w M_{\exp } f_{\exp }$.

(trade balance condition in ME, M3) 


\subsubsection{The Export Tax}

The Optimal Value of Export Tax. We want to show that the optimal value of $s$ is $\frac{\beta-\rho}{\beta}<1$. The market equilibrium conditions are the same as those in Section 2 with $\eta=1$ :

$$
\begin{gathered}
\text { (EXP) condition } A s^{\sigma} w^{1-\sigma}(\rho y)^{\sigma-1}=\sigma w f_{\exp }, \\
\text { (FE) condition } \quad(\theta-1) x^{-\beta}\left[f+m_{\exp } f_{\exp }\right]=\frac{f_{e}}{b^{\beta}}, \\
\text { (M) condition } M=\frac{1}{\sigma \theta\left(f+m_{\exp } f_{\exp }\right)}=\frac{(\theta-1) b^{\beta}}{\sigma \theta f_{e}} x^{-\beta}, \\
(\mathrm{TB}) \text { condition } M_{m} F_{\exp }=\frac{w}{s} M_{\exp } f_{\exp } \cdot \\
\text { (z) condition } 1=\sigma \theta M\left[f_{\exp } m_{\exp }+\frac{F_{\exp }}{w}\left(\gamma \frac{x}{w z}\right)^{\sigma-1}\right] .
\end{gathered}
$$

Now we are ready to prove that $s=\frac{\beta-\rho}{\beta}$ results in the maximal level of welfare.

Proof. Step 1. First, note that when $s$ rises, $y$ must fall and $x$ must rise. The proof is the same as in Section 6.3.2, with equations (43), (44), and (46) rewritten as

$$
(\mathrm{EXP}): w^{\sigma}=\frac{A \rho^{\sigma-1}}{\sigma f_{\exp }} s^{\sigma} y^{\sigma-1}, \quad \frac{F_{\exp }}{w}\left(\frac{\gamma x}{w z}\right)^{\sigma-1}=f, \quad \text { and } \quad w^{\sigma-\frac{\sigma-1}{\beta}} \propto\left(\frac{x}{y}\right)^{\sigma-1} \text {. }
$$

Step 2. This step is also the same as in Section 6.3 .2 with $\kappa(x)=\frac{w}{s} m_{x} \frac{f_{\exp }}{F_{\exp }}\left(\frac{F_{\exp } z}{f \gamma x}\right)^{\rho}$, and when we need to compare

$$
\frac{\beta}{\beta-\rho} \frac{f_{\exp }}{f}\left(\frac{x}{y}\right)^{\beta} \frac{1}{\kappa(x)} \text { versus } 1,
$$

from using (55) instead of (44) and the new $\kappa(x)$ function, we get:

$$
\begin{aligned}
& \frac{\beta}{\beta-\rho} \frac{\frac{f_{\exp }}{f}\left(\frac{x}{y}\right)^{\beta}}{\frac{w}{s} \frac{f_{\exp }}{F_{\exp }}\left(\frac{F_{\exp } z}{f \gamma x}\right)^{\rho}}=\frac{\beta}{\beta-\rho}\left(\frac{F_{\exp }}{f}\right)^{\frac{1}{\sigma}}\left(\frac{\gamma x}{z}\right)^{\rho} \frac{s}{w} \\
& =\frac{\beta}{\beta-\rho}\left(\frac{F_{\exp }}{f}\right)^{\frac{1}{\sigma}}\left(\frac{\gamma x}{z}\right)^{\rho} \frac{s}{\left(\frac{F_{\exp }}{f}\right)^{\frac{1}{\sigma}}\left(\frac{\gamma x}{z}\right)^{\rho}}=\frac{\beta}{\beta-\rho} s .
\end{aligned}
$$

Thus, the comparison above results in comparing $s$ with $\frac{\beta-\rho}{\beta}$, and we proved our results. 
First Best Allocation and Export Tax As in Section 6.3.2, it can be shown that the market equilibrium conditions for $s=\frac{\beta-\rho}{\beta}$ coincide with the system of equations (29)-(36), if

$$
\phi=R P^{\sigma-1}\left(\frac{\rho}{w}\right)^{\sigma}, \alpha=A\left(\frac{\rho}{w}\right)^{\sigma}\left(\frac{\beta-\rho}{\beta}\right)^{\sigma}, \lambda=R^{\rho-1} P^{-\rho} w, \zeta=\frac{\beta-\rho}{\beta} R^{\rho-1} P^{-\rho} .
$$

\subsubsection{The Import Tariff}

The Optimal Value of Import Tariff. The derivations of the equilibrium conditions with the import tariff $t$ are very similar to those in Section 2 with $\eta=1$ and $s=1$. As a result, we have

$$
\begin{aligned}
& (\mathrm{EXP}) \text { condition } A w^{1-\sigma}(\rho y)^{\sigma-1}=\sigma w f_{\exp } \text {, and } \\
& (\mathrm{FE}) \text { condition } \quad(\theta-1) x^{-\beta}\left[f+m_{\exp } f_{\exp }\right]=f_{e} / b^{\beta} . \\
& \text { (TB) condition } \quad M_{m} F_{\exp }=w M_{\exp } f_{\exp } .
\end{aligned}
$$

We need to derive the zero profit condition for importers. The demand for the foreign variety $v$ is $q_{m}(v)=R P^{\sigma-1}\left(t p_{m}(v)\right)^{-\sigma}$. The expenditures in the economy are $R=w+T$, where $T$ is:

$$
T=M_{m} \int_{z} \frac{(t-1)}{t}[t p(\varphi)] q_{m}(\varphi) \mu(\varphi) d \varphi=(t-1)\left[R P^{\sigma-1} t^{-\sigma}\right] \theta M_{m}\left(\frac{\rho z}{\gamma}\right)^{\sigma-1}
$$

where $P^{1-\sigma}=\theta\left[M_{m}\left(\frac{\rho z}{\gamma t}\right)^{\sigma-1}+M\left(\frac{\rho x}{w}\right)^{\sigma-1}\right]$. Then $R=\frac{w}{1-\frac{t-1}{t} P^{\sigma-1} \theta M_{m}\left(\frac{\rho z}{\gamma t}\right)^{\sigma-1}}$, and the new (z) condition can be written as

$$
\begin{aligned}
R P^{\sigma-1} \frac{1}{t}\left(\frac{\rho z}{\gamma t}\right)^{\sigma-1} & =\sigma F_{\exp } \Longleftrightarrow \frac{w \frac{1}{t}\left(\frac{\rho z}{\gamma t}\right)^{\sigma-1}}{P^{1-\sigma}-\frac{t-1}{t} \theta M_{m}\left(\frac{\rho z}{\gamma t}\right)^{\sigma-1}}=\sigma F_{\exp } \\
& \text { or } \frac{w}{\sigma \theta F_{\exp }}=M_{m}+M t^{\sigma}\left(\frac{\gamma x}{w z}\right)^{\sigma-1}
\end{aligned}
$$

or using the (TB) condition,

$$
\text { (z) condition } \frac{w}{\sigma \theta F_{\exp }}=M_{x} \frac{w f_{\exp }}{F_{\exp }}+M t^{\sigma}\left(\frac{\gamma x}{w z}\right)^{\sigma-1} \text {. }
$$

Finally,

$$
\text { (M) condition: } \quad M=\frac{1}{\sigma \theta\left(f+m_{\exp } f_{\exp }\right)}=\frac{(\theta-1) b^{\beta}}{\sigma \theta f_{e}} x^{-\beta} .
$$

Now we are ready to prove that an import tariff $t=\frac{\beta}{\beta-\rho}$ maximizes welfare.

Proof. Step 1. First, we prove that when $t$ rises, $x$ falls, while $y$ and $w$ rise.

From the (FE) condition, $x$ and $y$ must move in the opposite direction. Assume that $y$ falls. 
Then $x$ rises and from

$$
(\mathrm{EXP}): \frac{w^{\sigma}}{y^{\sigma-1}}=\frac{A \rho^{\sigma-1}}{\sigma f_{\exp }}
$$

$w$ must fall. On the other hand, from

$$
\begin{gathered}
(\mathrm{z})+(\mathrm{M}) \quad \Rightarrow \frac{w}{F_{\exp }}=\frac{1}{f+m_{\exp } f_{\exp }}\left[m_{\exp } \frac{w f_{\exp }}{F_{\exp }}+t^{\sigma}\left(\frac{\gamma x}{w z}\right)^{\sigma-1}\right], \quad \text { or } \\
\frac{w}{F_{\exp }}=\frac{w}{F_{\exp }}+\frac{1}{f+m_{\exp } f_{\exp }}\left[t^{\sigma}\left(\frac{\gamma x}{w z}\right)^{\sigma-1}-\frac{w f}{F_{\exp }}\right], \quad \text { or } \\
t^{\sigma}\left(\frac{\gamma x}{w z}\right)^{\sigma-1}=\frac{w f}{F_{\exp }} \text { or } w^{\sigma}=t^{\sigma} \frac{F_{\exp }}{f}\left(\frac{\gamma x}{z}\right)^{\sigma-1} .
\end{gathered}
$$

Again, from $(\mathrm{TB})$ condition, $z^{-1}=\left(\frac{f_{\exp }}{F_{\exp }} w M_{\exp }\right)^{\frac{1}{\beta}} \propto w^{\frac{1}{\beta}} y^{-1}$ so that

$$
w \propto\left[t^{\sigma}\left(\frac{x}{y}\right)^{\sigma-1}\right]^{\frac{1}{\sigma-\frac{\sigma-1}{\beta}}}
$$

and from (62), $w$ rises, which contradicts to the previous conclusion about $w$. Thus, we proved that $y$ cannot fall with an increase in $t$, and as $t$ rises, $y$ and $w$ rise as well, and $x$ falls.

Step 2. Now we are ready to derive the optimal import tariff. Note that

$$
U^{\rho}=\int_{z}^{\infty}\left[q_{m}(\varphi)\right]^{\rho} M_{m} \mu(\varphi) d \varphi+\int_{x}^{\infty} q(\varphi)^{\rho} M \mu(\varphi) d \varphi=\theta(\sigma-1)^{\rho}\left[M_{m}\left(\frac{F_{\exp }}{\gamma} z\right)^{\rho}+M(f x)^{\rho}\right]
$$

We will show that $U^{\rho}=d(x) h(x)$, where as $x$ rises, $d(x) \equiv \theta(\sigma-1)^{\rho} M(f x)^{\rho}$ falls faster than $h(x) \equiv \frac{M_{m}}{M}\left(\frac{F_{\exp } z}{f \gamma x}\right)^{\rho}+1$ rises, if $t<\frac{\beta}{\beta-\rho}$, and the opposite happens, if $t>\frac{\beta}{\beta-\rho}$. In other words, $U(x)$ falls with $x$, if $t<\frac{\beta}{\beta-\rho}$, and it rises with $x$, if $t>\frac{\beta}{\beta-\rho}$. Then since $d x / d t<0$,

$$
\frac{d U}{d t}=\frac{d U}{d x} \frac{d x}{d t}=\left\{\begin{array}{l}
>0, \text { if } t<\frac{\beta}{\beta-\rho} \\
<0, \text { if } t>\frac{\beta}{\beta-\rho}
\end{array}\right.
$$

and the utility reaches its maximum, when $t=\frac{\beta}{\beta-\rho}$.

We can compare the elasticities of $d(x)$ and $h(x)$ and show that $\varepsilon_{d}>0>\varepsilon_{h}$. Thus, the behavior of $U^{\rho}=d(x) h(x)$ depends on the comparison of absolute terms $\left|\varepsilon_{d}\right|$ and $\left|\varepsilon_{h}\right|$. First, note that $\varepsilon_{d(x)}=\rho-\beta<0$. In addition, from the (TB) condition,

$$
h(x)=w m_{x} \frac{f_{\exp }}{F_{\exp }}\left(\frac{F_{\exp } z}{f \gamma x}\right)^{\rho}+1=\kappa(x)+1,
$$


where from $w \propto y^{\rho}$ and $z^{-1} \propto w^{\frac{1}{\beta}} y^{-1}$

$$
\begin{gathered}
\kappa(x)=w m_{x} \frac{f_{\exp }}{F_{\exp }}\left(\frac{F_{\exp } z}{f \gamma x}\right)^{\rho} \propto\left(\frac{x}{y}\right)^{\beta-\rho}\left(y^{1-\frac{\rho}{\beta}}\right)^{\rho}=x^{\beta-\rho} y^{(\beta-\rho)\left(\frac{\rho}{\beta}-1\right)}, \text { and } \\
\varepsilon_{h(x)}=\frac{h^{\prime}(x)}{h(x)} x=\frac{\kappa^{\prime}(x)}{1+\kappa(x)} x=\frac{\kappa^{\prime}(x)}{\kappa(x)} x \frac{\kappa(x)}{1+\kappa(x)}=\varepsilon_{\kappa(x)} \frac{\kappa(x)}{1+\kappa(x)}, \\
\text { where } \varepsilon_{\kappa(x)}=(\beta-\rho)+(\beta-\rho)\left(\frac{\rho}{\beta}-1\right) \varepsilon_{y(x)}, \text { and } \\
\varepsilon_{h}=(\beta-\rho)\left(1+\left(1-\frac{\rho}{\beta}\right) \frac{f}{f_{\exp }}\left(\frac{y}{x}\right)^{\beta}\right) \frac{\kappa(x)}{\gamma+\kappa(x)}>0 .
\end{gathered}
$$

Finally, we can compare the absolute values of elasticities:

$$
\begin{gathered}
\left|\varepsilon_{d}\right|=\beta-\rho \text { versus }\left|\varepsilon_{h}\right|=(\beta-\rho)\left(1+\left(1-\frac{\rho}{\beta}\right) \frac{f}{f_{\exp }}\left(\frac{y}{x}\right)^{\beta}\right) \frac{\kappa(x)}{1+\kappa(x)} \text {, or } \\
\frac{\beta}{\beta-\rho} \frac{f_{\exp }}{f}\left(\frac{x}{y}\right)^{\beta} \frac{1}{\kappa(x)} \text { versus } 1 .
\end{gathered}
$$

To compare the left-hand side with 1 , we plug the expressions for $\kappa(x)$ and use $w^{\sigma}=t^{\sigma} \frac{F_{\exp }}{f}\left(\frac{\gamma x}{z}\right)^{\sigma-1}$ :

$$
\frac{\beta}{\beta-\rho} \frac{\frac{f_{\exp }}{f}\left(\frac{x}{y}\right)^{\beta}}{w m_{x} \frac{f_{\exp }}{F_{\exp }}\left(\frac{F_{\exp } z}{f \gamma x}\right)^{\rho}}=\frac{\beta}{\beta-\rho}\left(\frac{F_{\exp }}{f}\right)^{\frac{1}{\sigma}}\left(\frac{\gamma x}{z}\right)^{\rho} \frac{1}{w}=\frac{\beta}{\beta-\rho} \frac{1}{t} .
$$

Thus, we proved our results.

First Best Allocation and Import Tariff As in Section 6.3.2, it can be shown that the market equilibrium conditions for $t=\frac{\beta}{\beta-\rho}$ coincide with system of equations (29)-(36), if

$$
\phi=R P^{\sigma-1}\left(\frac{\rho}{w}\right)^{\sigma}, \quad \alpha=A\left(\frac{\rho}{w}\right)^{\sigma}, \lambda=R^{\rho-1} P^{-\rho} w, \quad \zeta=R^{\rho-1} P^{-\rho} .
$$

\subsection{Proof of Propositions 2 and 3}

The market equilibrium conditions for the case of the export subsidy in the presence of the consumption subsidy are derived in Section 3. Hereafter, we assume that the government has in place the optimal consumption subsidy (i.e., $\eta=\frac{\beta-\rho}{\beta}$ ) and explore how export subsidies affect the three components of the utility function by first proving Proposition 2 and then proving Proposition 3. Proof. The proofs for Propositions 2 and 3 are the same as in Section 6.3.3 with the comparison of $\varepsilon_{h(x)}$ and $\varepsilon_{d(x)}$ resulting in the comparison $s \gtreqless 1$. 


\subsection{Quantitative Exercise for Three Components of Utility Function}

We want to study the behavior of TOT and variety indices first. We show below that anything is possible, i.e., there are 3 cases: (Which case happens depends on the parameters.)

(1) TOT index falls, Variety index falls;

This happens if we set the parameters:

$$
\begin{aligned}
\beta & =4, \quad \sigma=3.8, \quad b=1, \\
\frac{f_{\exp }}{f} & =15000, \quad \frac{F_{\exp }}{f}=1500, \quad \frac{f_{e}}{f}=0.3, \quad \frac{A}{f}=200000, \quad \frac{L}{f}=0.5,
\end{aligned}
$$

and vary $s$ between 0.3 and 2.7. Then $\beta>\sigma, y>x>b, M_{e}>0$, wage $>0$, and both indices fall.

(2) TOT index falls, Variety index rises;

Compared to case (1), the only parameters needed to be changed to get such behavior of indices are $\frac{f_{\exp }}{f}=150000$ and $\frac{F_{\exp }}{f}=1.5$.

(3) TOT index rises, Variety index falls;

Again, compared to case (1), we change only 2 parameters: now $\frac{f_{\exp }}{f}=15$ and $\frac{F_{\exp }}{f}=15000$.

Finally, note that in the second case it can be shown that the curvature first falls and then starts to rise, so its behavior with respect to export subsidy is ambiguous as well. 